\title{
HYDRODYNAMIC SCALING LIMIT OF CONTINUUM SOLID-ON-SOLID MODEL
}

\author{
ANAMARIA SAVU \\ Received 1 June 2005; Revised 9 January 2006; Accepted 5 March 2006
}

\begin{abstract}
A fourth-order nonlinear evolution equation is derived from a microscopic model for surface diffusion, namely, the continuum solid-on-solid model. We use the method developed by Varadhan for the computation of the hydrodynamic scaling limit of nongradient models. What distinguishes our model from other models discussed so far is the presence of two conservation laws for the dynamics in a nonperiodic box and the complex dynamics that is not nearest-neighbor interaction. Along the way, a few steps have to be adapted to our new context. As a byproduct of our main result, we also derive the hydrodynamic scaling limit of a perturbation of the continuum solid-on-solid model, a model that incorporates both surface diffusion and surface electromigration.
\end{abstract}

Copyright (C 2006 Anamaria Savu. This is an open access article distributed under the Creative Commons Attribution License, which permits unrestricted use, distribution, and reproduction in any medium, provided the original work is properly cited.

\section{Introduction}

A process of great technological importance, molecular beam epitaxy (MBE), is used to manufacture computer chips and semiconductor devices, see Barabási and Stanley [1]. In general, the chip is constructed by spraying a beam of atoms on a flat surface. There are three phenomena that take place in the construction process, namely, deposition, diffusion, and desorption. The atoms arrive or deposit on the surface and do not stick on the first contact point, but diffuse or walk on the surface. When an atom reaches the edge of another wandering atom, the two atoms meet or glue together, forming islands. Sometimes an atom may jump out of the surface. Smaller islands may develop into larger islands affecting the roughness of the surface on the macroscopic scale. A rough surface does not have very good contact properties and engineers would like to understand the basic mechanisms affecting the morphology in general.

How deposition and desorption affect the morphology of the surface is quite well understood. It is of great importance to know how the profile of the surface evolves on the 
macroscopic scale if the atoms that make up the surface diffuse. We assume that no atoms arrive or leave the surface.

The present paper discusses a model for surface diffusion, the continuum solid-onsolid model, known also as the fourth-order Ginzburg-Landau model. The system has a complex interaction that is not a nearest-neighbor interaction and has two conserved quantities in a nonperiodic box, namely, the total slope of the surface and the linear mean of the surface slope. Under the assumption that the molecules of the surface follow the dynamics of the continuum solid-on-solid model, we will prove that the dynamics of the surface slope profile on the macroscale is a fourth-order nonlinear equation. Since the model is nongradient, the derivation of the limit is not trivial and we will use the method developed by Varadhan in [19] and further extended by Quastel in [13], and by Varadhan and Yau in [20]. Even though we have an interaction that is not of nearest-neighbor type, the microscopic current still writes as the sum of the Laplacian of the slope field and the fluctuations. Usually in nearest-neighbor models after subtracting the fluctuations from the current, we end up with the gradient of some local functions. We use the result that for continuum solid-on-solid model, the space of exact functions has codimension one inside the space of closed functions, see Savu $[16,17]$.

We also include a discussion of a perturbation of the continuum solid-on-solid model, where the evolution of the surface is driven by both diffusion and electric field. The electric field will add one extra second-order term to the final nonlinear equation.

The continuum solid-on-solid model is an approximation of the discrete solid-onsolid model, and hence is not considered a truly microscopic model. Unfortunately at the time of writing this paper, we did not have the required techniques to solve the discrete solid-on-solid model. However, partial rigorous results and numerical analysis are available for the discrete case, see Krug et al. [10].

Finally the paper is organized as follows. Section 2 contains the description of continuum solid-on-solid model and the description of the model for surface electromigration, the statement of the main results, and a note on similar models considered in the literature so far. Section 3 outlines the proof of the main result, the computation of the scaling limit of continuum solid-on-solid model. Section 4 shows how the final nonlinear equation is identified. In Section 5, we calculate the asymptotics of central limit variance, the main ingredient used in Section 4 to prove that the microscopic current can be replaced by a multiple of the field Laplacian. In Section 6, we calculate the scaling limit of the modified model to incorporate surface electromigration.

\section{The models}

The continuum solid-on-solid model is a one-dimensional lattice system with continuous-order parameter used to study the evolution of an interface. The model describes the movement of the interface at the mesoscopic level, and hence is not considered a truly microscopic model that captures all the aspects of the particle motion, but it has the advantage of being more suitable for computations.

The height model. Let $\mathbb{T}$ be the torus represented as the interval $[0,1]$ with 0 and 1 identified. For each positive integer $N$, there are $N$ scaled periodic lattice points located at sites 
$i / N$ in $\mathbb{T}, i=1, \ldots, N$. We will denote by $h_{i}(t)$ the height of the surface at the site $i / N$, at time $t$. Also because of the periodicity of the lattice points, $h_{N+1}(t)=h_{1}(t)$. The energy function $H_{N}(h)$ of a height configuration $h$ is chosen to be invariant under the global translation $h_{i}(t) \mapsto h_{i}(t)+c$ and has the form

$$
H_{N}(h)=\sum_{i=1}^{N} V\left(h_{i+1}-h_{i}\right) .
$$

In this paper, we will assume that the potential is quadratic $V(x)=x^{2}$. We will require the evolution in time of the surface to be conservative, in other words, to preserve the sum of the heights, to have as invariant measure the infinite-mass measure $e^{-H_{N}(h)} d h$, and to be reversible. All these properties are satisfied by the solution of the stochastic differential system:

$$
d h_{i}(t)=-\frac{N^{4}}{2}\left(w_{i}-w_{i-1}\right) d t+N^{2}\left(\sqrt{a_{i}} d B_{i}-\sqrt{a_{i-1}} d B_{i-1}\right), \quad 1 \leq i \leq N .
$$

Above, $a_{i}(h)=a\left(h_{i}-h_{i-1}, h_{i+1}-h_{i}, h_{i+2}-h_{i+1}\right)$, where $a$ is a function with bounded continuous first derivatives. Also $a$ satisfies $0<1 / a^{*} \leq a\left(x_{-1}, x_{0}, x_{1}\right) \leq a^{*}<\infty$. The $n$ copies of the Brownian motion $B_{i}, i=1, \ldots, n$, are independent. We define the instantaneous current $w_{i}(h)$ of particles over the bond $\{i, i+1\}$,

$$
\begin{aligned}
w_{i}(h)= & \left(\partial_{-1} a-2 \partial_{0} a+\partial_{1} a\right)\left(h_{i}-h_{i-1}, h_{i+1}-h_{i}, h_{i+2}-h_{i+1}\right) \\
& -a_{i}(h)\left(V^{\prime}\left(h_{i}-h_{i-1}\right)-2 V^{\prime}\left(h_{i+1}-h_{i}\right)+V^{\prime}\left(h_{i+2}-h_{i+1}\right)\right) .
\end{aligned}
$$

Because the height process preserves the sum of the heights of the surface, this dynamics models surface diffusion.

The slope process. A crucial property of the dynamics of the heights is the gauge property, namely, the dynamics invariance under the action of the group $G$ of translation in the $(1, \ldots, 1)$ direction,

$$
G=\left\{T: \mathbb{R}^{N} \longrightarrow \mathbb{R}^{N} \mid T\left(x_{1}, \ldots, x_{N}\right)=\left(x_{1}+c, \ldots, x_{N}+c\right), c \in \mathbb{R}\right\}
$$

Hence there exists an induced dynamics on the quotient space $\mathbb{R}^{N} / G$ of equivalence classes. A representative of an equivalence class is the slope configuration $x_{i}(t)=h_{i+1}(t)-$ $h_{i}(t), 1 \leq i \leq N-1$, and $x_{N}(t)=h_{1}(t)-h_{N}(t)$. Note that $\sum_{i=1}^{N} x_{i}(t)=0$.

As a function of the slope configuration of the surface, the energy becomes $H_{N}(x)=$ $\sum_{i=1}^{N} V\left(x_{i}\right)$.

In the sequel, we will study the slope process rather than the height process. The slope process is reversible and has as equilibrium distribution, the product probability measure $d \nu_{N}^{\text {eq }}=e^{-H_{N}(x)} / Z^{N} d x$. Below, we write down the stochastic differential system, the generator, and the Dirichlet form of the slope process:

$$
d x_{i}(t)=\frac{N^{4}}{2}\left(w_{i+1}-2 w_{i}+w_{i-1}\right) d t+N^{2}\left(\sqrt{a_{i-1}} d B_{i-1}-2 \sqrt{a_{i}} d B_{i}+\sqrt{a_{i+1}} d B_{i+1}\right),
$$




$$
\begin{aligned}
& N^{4} L_{N}(f)=\frac{N^{4}}{2} \sum_{i=1}^{N} a_{i}(x)\left(\partial_{i+1}-2 \partial_{i}+\partial_{i-1}\right)^{2} f+w_{i}(x)\left(\partial_{i+1}-2 \partial_{i}+\partial_{i-1}\right) f, \\
& N^{4} D_{N}(f)=\frac{N^{4}}{2} \int_{\mathbb{R}^{N}} \sum_{i=1}^{N} a_{i}(x)\left(\partial_{i+1} f-2 \partial_{i} f-\partial_{i-1} f\right)^{2}(x) \frac{e^{-H_{N}(x)}}{Z^{N}} d x .
\end{aligned}
$$

The factor $N^{4}$ in the generator with the lattice spacing of $1 / N$ represents the scaling of space and time. This scaling is needed to observe a nontrivial motion in the limit.

The diffusion (2.5) is driven in the direction of the linear vector fields $X_{i}=\partial_{i+1}-2 \partial_{i}+$ $\partial_{i-1}, 1 \leq i \leq N$, therefore is not ergodic in the whole space $\mathbb{R}^{N}$. It becomes ergodic when restricted to the hyperplane $x_{1}+\cdots+x_{N}=N \bar{x}$ of average slope $\bar{x}$. The unique equilibrium probability measure of the dynamics restricted to the hyperplane is the conditional probability $e^{-H_{N}(x)} / Z^{N} d x$ given that $x_{1}+\cdots+x_{N}=N \bar{x}$.

Instantaneously, the slope profile of the surface decreases at some site $i$ twice as much as it increases at the adjacent sites $i-1, i+1$. We see that any update of the slope configuration affects the slopes at three sites. This type of interaction, known as the three-site interaction, is quite complex and has been rarely studied, so far (see $[2,12])$.

We should note an integration-by-parts property of the current $w_{i}$ for all bounded and smooth functions $f$ :

$$
E^{\mathrm{eq}}\left[w_{i} \cdot f\right]=E^{\mathrm{eq}}\left[a_{i} \cdot\left(\partial_{i+1}-2 \partial_{i}+\partial_{i-1}\right) f\right] .
$$

The expected value above is with respect to the equilibrium measure $d \nu_{N}^{\mathrm{eq}}$.

The dynamics in a nomperiodic box. Although we are concerned with the study of the slope process defined on a periodic space, we make use of a similar slope process evolving in a box with nonperiodic boundary. Below, we describe this new dynamics and its main properties. Suppose $\Lambda$ is a box with finitely many sites of the lattice $\mathbb{Z}$. The infinitesimal generators

$$
L_{\Lambda}(f)=\frac{1}{2} \sum_{i \in \Lambda, i+1, i-1 \in \Lambda} a_{i}(x)\left(\partial_{i+1}-2 \partial_{i}+\partial_{i-1}\right)^{2} f+w_{i}(x)\left(\partial_{i+1}-2 \partial_{i}+\partial_{i-1}\right) f,
$$

respectively,

$$
L_{\infty}(f)=\frac{1}{2} \sum_{i \in \mathbb{Z}} a_{i}(x)\left(\partial_{i+1}-2 \partial_{i}+\partial_{i-1}\right)^{2} f+w_{i}(x)\left(\partial_{i+1}-2 \partial_{i}+\partial_{i-1}\right) f
$$

produce two diffusion processes.

In the case of the first dynamics, generated by $L_{\Lambda}$, there is no transport of mass over the boundary of the box, so the box does not have periodic boundary. If the box $\Lambda=[-l, l] \cap \mathbb{Z}$ or $\Lambda=[i-l, i+l] \cap \mathbb{Z}$, we use the shorter notation $L_{l}$, respectively, $L_{i, l}$ for the generator $L_{\Lambda}$. The second dynamics generated by $L_{\infty}$ is a dynamics on the infinite lattice $\mathbb{Z}$. The dynamics $L_{i, l}$ preserves the average slope $y_{i, l}^{1}=\left(x_{i-l}+\cdots+x_{i+l}\right) /(2 l+1)$, and the linear mean of the slopes $y_{i, l}^{2}=\left((-l) x_{i-l}+\cdots+l x_{i+l}\right) / l(l+1)$ inside the box $[i-l, i+l] \cap \mathbb{Z}$. The second conserved quantity $y_{i, l}^{2}$ should be understood as a boundary condition that 
is preserved in time. The linear mean slope $y_{i, l}^{2}$ is conserved in time because we have a model for surface diffusion and the total height of the surface does not change as time passes by. We will use two different notations $\bar{x}_{i, l}$ and $y_{i, l}^{1}$ for the mean slope of the field in the box centered at $i$, of size $l$. Also $y_{i, l}$ stands for the vector of the conserved quantities, $\left(y_{i, l}^{1}, y_{i, l}^{2}\right)$. As a convention, we will drop the subscript $i$, meaning the center of the box, if the box is centered at the origin.

Equilibrium measures. We proceed to describe next the equilibrium measures of the dynamics that we have introduced. For the dynamics $L_{i, l}$, the grand canonical measure is the product probability measure $v_{\alpha, i, l}^{\mathrm{gc}}=\bigotimes_{j=i-l}^{i+l}\left(e^{\alpha x_{j}-V\left(x_{j}\right)} / Z(\alpha)\right) d x_{j}$, whereas the canonical measure is the conditional probability measure $v_{y, i, l}^{c}=v_{\alpha, i, l}^{\mathrm{gc}}\left(\cdot \mid y_{i, l}\right)$ given the level set

$$
\left\{x \in \mathbb{R}^{2 l+1} \mid \frac{x_{i-l}+\cdots+x_{i+l}}{2 l+1}=y_{i, l}^{1}, \frac{(-l) x_{i-l}+\cdots+l x_{i+l}}{l(l+1)}=y_{i, l}^{2}\right\} .
$$

The canonical measure is the unique stationary probability measure for the restricted dynamics on this set. The Dirichlet forms of the operators $L_{l}, L_{i, l}$, with respect to the grand canonical measure with $\alpha=0$, are denoted by $D_{l}(f)$, respectively, $D_{i, l}(f)$. The Dirichlet form of the operator $L_{l}$ with respect to the canonical measure is denoted by $D_{\gamma_{y, l}^{c}}(f)$. For $L_{\infty}$, the product measures $\nu_{\alpha}^{\mathrm{gc}}=\bigotimes_{i \in \mathbb{Z}}\left(e^{\alpha x_{i}-V\left(x_{i}\right)} / Z(\alpha)\right) d x_{i}$ are equilibrium measures. We will use the notation $\nu_{N}^{\text {eq }}$ for the product probability measure $\bigotimes_{i=1}^{N}\left(e^{-V\left(x_{i}\right)} / Z\right) d x_{i}$.

A model for surface electromigration. We consider also a perturbation of the continuum solid-on-solid model. The new system describes the evolution of a one-dimensional surface driven by both surface diffusion and surface electromigration. The surface electromigration refers to the motion of atoms on a solid surface that is caused by an electric current in the material. The electric field interacts with the atoms of the surface as the wind blows the sand particles, and a ripple pattern is observed in the long run. Electromigration along interfaces is believed to play a crucial role in the failure of metallic circuits, see Schimschak and Krug [18] for further details.

We assume the electric field is a continuous function $E(t, \theta)$ defined on $[0, T] \times \mathbb{T}$. As before, $x_{i}$ represents the slope of the surface at the site $i / N$. The generator of the system on a periodic lattice, that incorporates the action of the electric field, is

$$
N^{4} L_{N, E}(f)=N^{4} L_{N}(f)+\frac{N^{2}}{2} \sum_{i=1}^{N} E\left(t, \frac{i}{N}\right) a\left(x_{i-1}, x_{i}, x_{i+1}\right)\left(\partial_{i-1}-2 \partial_{i}+\partial_{i+1}\right) f .
$$

Hydrodynamic scaling limit of the models. We will call $P_{N, T}^{\text {neq }}$ and $P_{N, T}^{\text {eq }}$ the law up to time $T$ of the slope process (2.5) started in some nonequilibrium distribution $\nu_{N}^{\text {neq }}$ and equilibrium distribution $\nu_{N}^{\text {eq }}$, respectively. The law up to time $T$ of the slope process (2.12), driven by the electric field, started in the nonequilibrium measure $\nu_{N}^{\text {neq }}$ will be called $P_{N, E, T}^{\text {neq }}$.

Under $P_{N, T}^{\text {neq }}$ and $P_{N, T}^{\text {eq }}$ the random variable

$$
\pi_{N}(t)=\frac{1}{N}\left(x_{1}(t) \delta_{1 / N}+\cdots+x_{N}(t) \delta_{N / N}\right)
$$


has distributions $Q_{N, T}^{\text {neq }}$ and $Q_{N, T}^{\mathrm{eq}}$, respectively. We also refer to the random variable (2.13) as the empirical distribution. Every realization of this random variable is a measurevalued continuous path and $Q_{N, T}^{\text {neq }}$ is a distribution on the space $\mathscr{X}=\bigcup_{(l \geq 0)} C\left([0, T], M_{l}\right)$. The space $\mathscr{X}$ is endowed with the inductive limit topology, the strongest topology that makes all the inclusions of $C\left([0, T], M_{l}\right)$ continuous. The space of signed measures $M_{l}$, with total variation not exceeding $l$, is a metrizable space with the weak topology.

We say that a model has hydrodynamic scaling limit if under certain assumptions, the sequence of laws of empirical distributions has a limit that is supported on the solution of an initial value problem.

Definition 2.1. A sequence of initial distributions $\nu_{N}^{\text {neq }}$ on $\mathbb{R}^{N}$ is said to correspond to the macroscopic slope profile $m_{0} \in L^{1}(\mathbb{T})$ if the random variable $\pi_{N}$ converges weakly in probability to $\delta_{m_{0}(\theta) d \theta}$, that is, for any continuous function $\phi \in C(\mathbb{T})$ and any $\epsilon>0$,

$$
\limsup _{N \rightarrow \infty} v_{N}^{\text {neq }}\left\{\left|\frac{1}{N} \sum_{i=1}^{N} \phi\left(\frac{i}{N}\right) x_{i}-\int_{\mathbb{T}} \phi(\theta) m_{0}(\theta) d \theta\right|>\epsilon\right\}=0
$$

Our first result of the paper says that the continuum solid-on solid model has a scaling limit and provides the form of the limiting evolution equation. More precisely, we have the following theorem.

Theorem 2.2. Assume that the potential $V(x)$ is equal to $x^{2} / 2$. Let $m_{0} \in L^{1}(\mathbb{T})$ be a macroscopic slope profile such that $\int_{\mathbb{T}} m_{0}(\theta) d \theta=0$. Assume that the sequence of initial distributions $\left\{\nu_{N}^{\text {neq }}\right\}_{N}$ corresponds to the profile $m_{0}$ and the initial relative entropy $H\left(\nu_{N}^{\text {neq }} \mid \nu_{N}^{\text {eq }}\right)$ is of order $\mathcal{O}(N)$. Then the sequence of probability measures $\left\{Q_{N, T}^{\text {neq }}\right\}_{N \geq 0}$ is tight in $\mathscr{X}$.

Any possible limit $Q_{T}$ of a convergent subsequence of $\left\{Q_{N, T}^{\text {neq }}\right\}_{N \geq 0}$ is concentrated on the weak solutions $(m(t, \theta) d \theta)_{t \in[0, T]}$ of the Cauchy problem with periodic boundary conditions

$$
\partial_{t} m=-\frac{1}{2} \partial_{\theta}^{2}\left(\hat{a}(m) \partial_{\theta}^{2} m\right), \quad m(0, \theta)=m_{0}(\theta), \quad \theta \in \mathbb{T} .
$$

The transport coefficient $\hat{a}$ is a nonrandom continuous function on $\mathbb{R}$, given by the following variational formula:

$$
\hat{a}(\alpha)=\inf _{g} E_{\gamma_{\alpha}^{\mathrm{gc}}}\left[a\left(x_{-1}, x_{0}, x_{1}\right)\left(1+\left(\partial_{i+1}-2 \partial_{i}+\partial_{i-1}\right)\left(\sum_{j \in \mathbb{Z}} \tau^{j} g\right)\right)^{2}\right] .
$$

The infimum on the line above is taken over all local functions $g\left(x_{-s}, \ldots, x_{s}, \bar{y}_{0, l}^{1}\right)$ of the slope configuration. The shift $\tau^{j}$ acts on configurations $\left(\tau^{j} x\right)_{k}=x_{k+j}$ and on local functions, $\left(\tau^{j} g\right)(x)=g\left(\tau^{j} x\right)$. The expectation $E_{\gamma_{\alpha}^{\mathrm{gc}}}$ is with respect to the grand canonical measure $\nu_{\alpha}^{\mathrm{gc}}$.

Note. It can be shown that the transport coefficient $\hat{a}$ is a continuous and bounded above and below function (see Kipnis and Landim [9]). Also we expect that the methods of Landim et al. [11] can show that $\hat{a}$ is smooth, but we will not pursue it here.

Note. By a weak solution of the Cauchy problem (2.15) we mean a path $m \in \mathscr{X}$ such that for each time $T \geq 0$, the value of the path $m$ at the time $T$ is a Lebesgue absolutely 
continuous measure on $\mathbb{T}$, satisfying the energy estimate

$$
\int_{0}^{T} \int_{\mathbb{T}}\left(\partial_{\theta}^{2} m(t, \theta)\right)^{2} d \theta d t<\infty
$$

Moreover for each $0 \leq T<\infty$ and for each test function $\phi \in C^{1,2}([0, T] \times \mathbb{T})$,

$$
\begin{gathered}
\int_{\mathbb{T}} m(T, \theta) \phi(T, \theta) d \theta-\int_{\mathbb{T}} m(0, \theta) \phi(0, \theta) d \theta-\int_{0}^{T} \int_{\mathbb{T}} m(s, \theta) \partial_{s} \phi(s, \theta) d \theta d s \\
+\frac{1}{2} \int_{0}^{T} \int_{\mathbb{T}} \hat{a}(m) \partial_{\theta}^{2} m(s, \theta) \partial_{\theta}^{2} \phi(s, \theta) d \theta d s=0 .
\end{gathered}
$$

If we assume that the initial condition $m_{0}$ has the property that $\int_{\mathbb{T}} m_{0}(\theta) d \theta=0$, then for each time $t \geq 0$, the solution satisfies $\int_{\mathbb{T}} m(t, \theta) d \theta=0$.

Uniqueness of weak solutions of the Cauchy problem, which satisfy the energy estimate (2.17), has not been proved yet. If the transport coefficient $\hat{a}$ does not depend on the field $m$, the uniqueness of the Cauchy problem is known and can be found in Eidel'man's book [5].

As will be explained later, the fluctuation-dissipation equation for the continuum solid-on-solid model follows from the direct sum decomposition of a Hilbert space to be defined next.

We define the Hilbert space of closed functions $\mathscr{C}_{X}$ to be the space of those $\xi \in L^{2}\left(d v_{\alpha}^{\mathrm{gc}}\right)$ that satisfy in the weak sense the equations $X_{i}\left(\tau^{j} \xi\right)=X_{j}\left(\tau^{i} \xi\right)$ for all integers $i$ and $j$. It is not hard to see that a subspace of $\mathscr{C}_{X}$ is the closed linear span in $L^{2}\left(d v_{\alpha}^{\mathrm{gc}}\right)$ of functions $\xi_{g}=$ $X_{0}\left(\sum_{j \in \mathbb{Z}} \tau^{j} g\right)$, where $g$ is a bounded local function with bounded first derivatives. Even though the infinite sum $\sum_{j \in \mathbb{Z}} \tau^{j} g$ does not make sense, the function $\xi_{g}$ is well defined because the vector field kills all but finitely many terms of the infinite sum. We will call this space the space of exact functions and we will use the notation $\mathscr{E}_{X}$. The space of exact functions has codimension one inside the space of closed functions. We include below the statement of this result.

Lemma 2.3. Let 1 denote the constant function 1 . The direct sum decomposition holds:

$$
\mathscr{C}_{X}=\mathbb{R} 1 \bigoplus \mathscr{E}_{X}
$$

In this paper, we do not include the proof of this result, since it is the subject of the paper by Savu [17]. For the proof of Lemma 2.3, it is important that the potential $V(x)$ is quadratic and is equal to $x^{2} / 2$.

The second result of the present paper proves that the model for surface electromigration (2.12) has a scaling limit as well, and calculates the limiting evolution equation.

Theorem 2.4. Suppose the hypotheses of Theorem 2.2 is satisfied. Then the sequence of probability measures $\left\{Q_{N, E, T}^{\text {neq }}\right\}_{N \geq 0}$ is tight in $\mathscr{X}$, and any possible limit $Q_{E, T}$ is supported on the weak solutions of the Cauchy problem

$$
\partial_{t} m=-\frac{1}{2} \partial_{\theta}^{2}\left(\hat{a}(m)\left(\partial_{\theta}^{2} m+E\right)\right), \quad m(0, \theta)=m_{0}(\theta), \quad \theta \in \mathbb{T} .
$$


The transport coefficient $\hat{a}$ is given by the same variational formula as in the statement of Theorem 2.2.

Similar models. The continuum solid-on-solid model belongs to a large class of Ginzburg-Landau models. The slope model, considered in this paper, is of nongradient type and has an unusual dynamics because two neighboring exchanges occur always simultaneously. Nishikawa [12], and Bertini et al. [2] have investigated the hydrodynamic scaling limit even in higher dimension of the gradient version of the slope model. They have found that on the macroscopic scale, the interface follows a fourth-order nonlinear evolution equation. The nongradient model considered in this paper is more accurate and captures more features of the physical phenomenon than the gradient models discussed in $[2,12]$. Our model is nongradient because the microscopic mobility $a$ defining the dynamics (2.2) is assumed to depend on the height configuration; an assumption that is more realistic from a physical point of view than the assumption of $[2,12]$ that $a$ is constant and independent of the height configuration.

Another similar model, the second-order Ginzburg-Landau model for a one-dimensional configuration, where the sum of the heights is not conserved, was the subject of extensive discussions in the literature: the hydrodynamic scaling limit was derived by Fritz [6], Guo et al. [8] for the gradient version, and by Varadhan [19] for the nongradient case, whereas the nonequilibrium fluctuations have been proved by Chang and Yau [3].

We also restrict our attention to the one-dimensional solid-on-solid model, since a corresponding dynamics on a multidimensional interface is much more difficult to be treated, already in the nonconservative case (according to the work of Funaki and Spohn [7]).

The second-order Ginzburg-Landau model and the continuum solid-on-solid model correspond to Glauber, respectively, Kawasaki dynamics in the context of interacting particle systems. As expected, a different dynamics at the mesoscopic level causes different dynamics at the macroscopic level, a second-order parabolic differential equation in the case of second-order Ginzburg-Landau model versus a fourth-order parabolic differential equation for the continuum solid-on-solid model.

\section{Hydrodynamic scaling limit of continuum solid-on-solid model}

In this section, we give a sketch of the main result, Theorem 2.2. We follow a standard scheme to derive the hydrodynamic scaling limit of the continuum solid-on-solid model. The existence of the limit follows from the tightness of the sequence of probability measures $\left\{Q_{N, T}^{\text {neq }}\right\}_{N}$. Let $Q_{T}$ be the limit of some weakly convergent subsequence of $\left\{Q_{N, T}^{\text {neq }}\right\}_{N}$. We proceed to characterize the limit $Q_{T}$, showing that it is supported on continuous paths with a certain regularity property, known as the energy estimate (2.17). The most involved part of the argument is the identification of the possible weak limit $Q_{T}$ as some probability measure supported on the weak solution of the Cauchy problem (2.15). We will make the assumption that the sequence of initial distributions $\left\{v_{N}^{\text {neq }}\right\}_{N}$ corresponds to some macroscopic profile $m_{0} \in L^{1}(\mathbb{T})$. 
Note on notations. Throughout the paper, we will make use of the shorter notation $\limsup \operatorname{sul}_{z_{1} \rightarrow i_{1}, \ldots, z_{n} \rightarrow i_{n}} f\left(z_{1}, \ldots, z_{n}\right)$ for the sequence of limits $\limsup {z_{1} \rightarrow i_{1}}_{\cdots} \cdots \limsup {z_{n} \rightarrow i_{n}}_{n} f\left(z_{1}\right.$, $\left.\ldots, z_{n}\right)$. If the limit points $i_{1}, \ldots, i_{n}$ are all equal to $i$ we use the shorter notation $\limsup z_{z_{1}, \ldots, z_{n} \rightarrow i} f\left(z_{1}, \ldots, z_{n}\right)$ for the sequence of limits $\limsup \sup _{z_{1} \rightarrow i} \cdots \limsup z_{n} \rightarrow i f\left(z_{1}\right.$, $\left.\ldots, z_{n}\right)$.

Tightness. As a consequence of Prohorov theorem and Arzela-Ascoli theorem, the tightness of the sequence $Q_{N, T}^{\text {neq }}$ follows from the next two lemmas.

Lemma 3.1. For any test function $\phi \in C^{2}(\mathbb{T})$, any finite time $T$, and any $\epsilon>0$,

$$
\limsup _{\delta \rightarrow 0, N \rightarrow \infty} P_{N, T}^{\text {neq }}\left\{\sup _{|s-t| \leq \delta, 0 \leq s, t \leq T}\left|\frac{1}{N} \sum_{i=1}^{N} \phi\left(\frac{i}{N}\right) x_{i}(t)-\frac{1}{N} \sum_{i=1}^{N} \phi\left(\frac{i}{N}\right) x_{i}(s)\right|>\epsilon\right\}=0 .
$$

LEMMA 3.2. The following convergence holds:

$$
\limsup _{l \rightarrow \infty, N \rightarrow \infty} P_{N, T}^{\text {neq }}\left\{\sup _{0 \leq t \leq T} \frac{1}{N} \sum_{i=1}^{N}\left|x_{i}(t)\right|>l\right\}=0 .
$$

It is interesting to note that the stronger superexponential estimates can be established for the process in equilibrium,

$$
\begin{gathered}
\limsup _{\delta \rightarrow 0, N \rightarrow \infty} \frac{1}{N} \log P_{N, T}^{\mathrm{eq}}\left\{\sup _{0 \leq t, s \leq T,|t-s| \leq \delta}\left|\int_{s}^{t} \frac{1}{N} \sum_{i=1}^{N} N^{2} w_{i}(u) \phi\left(\frac{i}{N}\right) d u\right| \geq \epsilon\right\}=-\infty, \\
\limsup _{l \rightarrow \infty, N \rightarrow \infty} \frac{1}{N} \log P_{N, T}^{\mathrm{eq}}\left\{\sup _{0 \leq t \leq T} \frac{1}{N} \sum_{i=1}^{N}\left|x_{i}(t)\right| \geq l\right\}=-\infty .
\end{gathered}
$$

The techniques used to prove Lemmas 3.1 and 3.2 are not new, and they can be used for a fairly large class of models (see Kipnis and Landim [9] or Guo et al. [8]), however they need to be adapted for the model taken into consideration in the present paper. For the reader's convenience, we include the proofs for Lemmas 3.1 and 3.2. Before giving the proofs of Lemmas 3.1 and 3.2, we remind the reader with the helpful inequalities: the Garsia-Rodemich-Rumsey inequality (3.4) and the entropy inequality (3.5).

LEMma 3.3 (Garsia-Rodemich-Rumsey inequality). Given a function $f$ and a strictly increasing function $\psi$ such that $\psi(0)=0$ and $\lim _{u \rightarrow \infty} \psi(u)=\infty$, then

$$
\begin{gathered}
\sup _{|t-s| \leq \delta, 0 \leq t, s \leq T}|f(t)-f(s)| \leq 8 \int_{0}^{\delta} \psi^{-1}\left(\frac{4 B}{u^{2}}\right) \frac{d u}{\sqrt{u}} \\
\text { where } B=\iint_{0}^{T} \psi\left(\frac{|f(t)-f(s)|}{\sqrt{t-s}}\right) d t d s .
\end{gathered}
$$

We also remind the reader that given two probability measures $\nu$ and $\mu$ on the same probability space such that $\nu$ is absolutely continuous with respect to $\mu$, the relative entropy of $\nu$ with respect to $\mu$ is defined by $H(\nu \mid \mu)=E_{\mu}[(d \nu / d \mu) \log (d \nu / d \mu)]$, where $d \nu / d \mu$ 
is the Radon-Nikodym derivative of $\nu$ relative to $\mu$. The entropy $H(\nu \mid \mu)$, always a positive quantity, is the optimal constant that makes the entropy inequality

$$
E_{\nu}[f] \leq \frac{1}{\alpha}\left\{H(\nu \mid \mu)+\log E_{\mu}\left[e^{\alpha f}\right]\right\}
$$

true for any bounded, measurable function $f$ and $\alpha>0$. A trivial consequence of the entropy inequality (3.5) helps us to estimate $v(A)$, where $A$ is some event,

$$
\nu(A) \leq \frac{\log (2)+H(\nu \mid \mu)}{\log (1+1 / \mu(A))} .
$$

Proof of Lemma 3.1. First we give an upper bound for the uniform modulus of continuity $m c(\delta)$ of the path $\left\{\sum_{i=1}^{N} \phi(i / N) x_{i}(t)\right\}_{0 \leq t \leq T}$,

$$
\begin{aligned}
m c(\delta)= & \sup _{|s-t| \leq \delta, 0 \leq s, t \leq T}\left|\frac{1}{N} \sum_{i=1}^{N} \phi\left(\frac{i}{N}\right) x_{i}(t)-\frac{1}{N} \sum_{i=1}^{N} \phi\left(\frac{i}{N}\right) x_{i}(s)\right| \\
\leq & \sup _{|s-t| \leq \delta, 0 \leq s, t \leq T}\left|\frac{N^{2}}{N} \int_{s}^{t} \sum_{i=1}^{N} N^{2}\left(\phi\left(\frac{i-1}{N}\right)-2 \phi\left(\frac{i}{N}\right)+\phi\left(\frac{i+1}{N}\right)\right) w_{i} d u\right| \\
& +\sup _{|s-t| \leq \delta, 0 \leq s, t \leq T}\left|M_{N}(t)-M_{N}(s)\right|,
\end{aligned}
$$

where $\left\{M_{N}(t)\right\}_{t>0}$ is the martingale,

$$
M_{N}(t)=N \sum_{i=1}^{N}\left(\phi\left(\frac{i-1}{N}\right)-2 \phi\left(\frac{i}{N}\right)+\phi\left(\frac{i+1}{N}\right)\right) \sqrt{a_{i}} B_{i}(t) .
$$

The uniform modulus of continuity of the martingale path $\left\{M_{N}(t)\right\}_{0 \leq t \leq T}$ converges to zero in probability since

$$
\begin{aligned}
& P_{N, T}^{\text {neq }}\left\{\sup _{|s-t| \leq \delta, 0 \leq s, t \leq T}\left|M_{N}(t)-M_{N}(s)\right|>\epsilon\right\} \\
& \quad \leq P_{N, T}^{\text {neq }}\left\{2 \sup _{0 \leq t \leq T}\left|M_{N}(t)\right|>\epsilon\right\} \leq \frac{4 E^{\text {neq }}\left[M_{N}^{2}(T)\right]}{\epsilon^{2}},
\end{aligned}
$$

and $\lim _{N \rightarrow \infty} E^{\text {neq }}\left[M_{N}^{2}(T)\right]=0$, see (4.9).

Since the test function $\phi$ has two bounded derivatives, for large values of $N, N^{2}(\phi((i-$ $1) / N)-2 \phi(i / N)+\phi((i+1) / N))$ is an approximation for $\phi^{\prime \prime}$. For the remaining part of the proof, we relabel $\phi^{\prime \prime}$ by $\phi$ and we assume that the test function $\phi$ is just continuous. It remains to show that

$$
\limsup _{\delta \rightarrow 0, N \rightarrow \infty} P_{N, T}^{\text {neq }}\left\{\sup _{0 \leq t, s \leq T,|t-s| \leq \delta}\left|\int_{s}^{t} \frac{1}{N} \sum_{i=1}^{N} N^{2} w_{i}(u) \phi\left(\frac{i}{N}\right) d u\right| \geq \epsilon\right\}=0 .
$$


Note that the entropy inequality (3.6) implies that (3.10) follows from

$$
\limsup _{\delta \rightarrow 0, N \rightarrow \infty} \frac{1}{N} \log P_{N, T}^{\mathrm{eq}}\left\{\sup _{0 \leq t, s \leq T, t-s \leq \delta}\left|\int_{s}^{t} \frac{1}{N} \sum_{i=1}^{N} N^{2} w_{i}(u) \phi\left(\frac{i}{N}\right) d u\right| \geq \epsilon\right\}=-\infty .
$$

We can choose the function $\psi$ to be $\exp (N u)-1$, in the Garsia-Rodemich-Rumsey inequality (3.4), to obtain

$$
\begin{aligned}
\sup _{|t-s| \leq \delta, 0 \leq t, s \leq T}|f(t)-f(s)| & \leq \frac{8}{N} \int_{0}^{\delta} \log \left(1+\frac{4 B}{u^{2}}\right) \frac{d u}{\sqrt{u}} \\
& \leq \frac{C}{N}\left(\sqrt{\delta} \log \left(\delta^{2}+4 B\right)+\sqrt{\delta}(1-\log (\delta))\right) .
\end{aligned}
$$

Because of (3.12), the proof of (3.11) is reduced to

$$
\lim _{N \rightarrow \infty} \frac{1}{N} \log \left[\iint_{0}^{T} E^{\mathrm{eq}}\left[\exp \left(\frac{N^{2}}{\sqrt{t-s}}\left|\int_{s}^{t} \sum_{i=1}^{N} w_{i}(u) \phi\left(\frac{i}{N}\right) d u\right|\right)\right] d t d s\right]<\infty,
$$

and to

$$
E^{\mathrm{eq}}\left[\exp \left(\left|\int_{s}^{t} N^{2} \sum_{i=1}^{N} w_{i}(u) \phi\left(\frac{i}{N}\right) d u\right|\right)\right] \leq 2 \exp \left(C(t-s) \sum_{i=1}^{N} \phi^{2}\left(\frac{i}{N}\right)\right),
$$

where $C$ is a constant independent of $\phi$. The inequality (3.14) follows from Lemma 3.4.

Lemma 3.4. There exists a constant $C$ independent of $\phi$ such that

$$
E^{\mathrm{eq}}\left[\exp \left(\left|\int_{s}^{t} N^{2} \sum_{i=1}^{N} w_{i}(u) \phi\left(\frac{i}{N}\right) d u\right|\right)\right] \leq 2 \exp \left(C(t-s) \sum_{i=1}^{N} \phi^{2}\left(\frac{i}{N}\right)\right) .
$$

Proof. The Feynman-Kac formula implies that

$$
\begin{aligned}
& E^{\mathrm{eq}}\left[\exp \left(\int_{s}^{t} N^{2} \sum_{i=1}^{N} w_{i}(u) \phi\left(\frac{i}{N}\right) d u\right)\right] \\
& \quad \leq \exp \left((t-s) \underset{L^{2}\left(\nu_{N}^{\text {eq }}\right)}{\operatorname{supspec}}\left(N^{4} L_{N}+N^{2} \sum_{i=1}^{N} w_{i}(u) \phi\left(\frac{i}{N}\right)\right)\right) .
\end{aligned}
$$


Moreover, we have the following eigenvalue estimate:

$$
\begin{aligned}
& \underset{\substack{L^{2}\left(\nu_{N}^{\text {eq }}\right) \\
\text { supspec }}}{ }\left[N^{4} L_{N}+N^{2} \sum_{i=1}^{N} w_{i}(u) \phi\left(\frac{i}{N}\right)\right] \\
& =\sup _{\rho, E^{\mathrm{eq}}\left[\rho^{2}\right]=1} N^{2} \sum_{i=1}^{N} \phi\left(\frac{i}{N}\right) E^{\mathrm{eq}}\left[a_{i} X_{i}\left(\rho^{2}\right)\right]-\frac{N^{4}}{2} D_{N}(\rho) \\
& \leq \sup _{\rho, E^{\mathrm{eq}}\left[\rho^{2}\right]=1} N^{2} \sum_{i=1}^{N}\left[\frac{A}{2} \phi\left(\frac{i}{N}\right)^{2} E^{\mathrm{eq}}\left[a_{i} \rho^{2}\right]+\frac{1}{2 A} E^{\mathrm{eq}}\left[a_{i}\left(X_{i}(\rho)\right)^{2}\right]\right]-\frac{N^{4}}{2} D_{N}(\rho) \\
& \leq C \sum_{i=1}^{N} \phi\left(\frac{i}{N}\right)^{2}, \quad \text { if we choose } A=\frac{1}{N^{2}} .
\end{aligned}
$$

Thus, the lemma follows.

Proof of Lemma 3.2. This lemma will follow from estimates on the moment-generating function of hitting time of the diffusion process (2.5) and the entropy inequality (3.6).

Let us call $A$ the event $\left\{\sup _{0 \leq t \leq T}(1 / N) \sum_{i=1}^{N}\left|x_{i}(t)\right| \geq l\right\}$. The entropy inequality (3.6) applied for $P_{N, T}^{\text {neq }}$ versus $P_{N, T}^{\text {eq }}$ gives us a bound for $P_{N, T}^{\text {neq }}(A)$,

$$
P_{N, T}^{\text {neq }}(A) \leq \frac{\log (2)+H\left(\nu_{N}^{\text {neq }} \mid \nu_{N}^{\text {eq }}\right)}{\log \left(1+1 / P_{N, T}^{\mathrm{eq}}(A)\right)}
$$

therefore (3.2) follows from

$$
\limsup _{l \rightarrow \infty, N \rightarrow \infty} \frac{1}{N} \log P_{N, T}^{\mathrm{eq}}\left\{\sup _{0 \leq t \leq T} \frac{1}{N} \sum_{i=1}^{N}\left|x_{i}(t)\right| \geq l\right\}=-\infty .
$$

For the process $\left\{x^{N}(t)\right\}_{t>0}$, we define $\sigma$ to be the first hitting time of the set

$$
\left\{x \in \mathbb{R}^{N}\left|\frac{1}{N} \sum_{i=1}^{N}\right| x_{i} \mid \geq l\right\}
$$

Choose some $\rho<1$ and define the function $f(x)=E_{x}\left[\rho^{\sigma}\right]$, which has the properties that $f(x)=1$ for $x \in A$ and $\rho(L f)(x)=(1-\rho) f(x)$ for $x \in A^{c}$.

It is sufficient to assume $T=1$. Chebyshev's inequality implies that

$$
P_{N, T}^{\mathrm{eq}}(A)=P_{N, T}^{\mathrm{eq}}(\sigma \leq 1) \leq \frac{1}{\rho} E_{\gamma_{N}^{\mathrm{eq}}}\left[\rho^{\sigma}\right] \leq \frac{1}{\rho}\left(E_{\nu_{N}^{\mathrm{eq}}}\left[f^{2}\right]\right)^{1 / 2} \leq \frac{1}{\rho}\left(E_{\gamma_{N}^{\mathrm{eq}}}\left[f^{2}\right]+\frac{\rho}{1-\rho} D_{\nu_{N}^{\mathrm{eq}}}(f)\right)^{1 / 2}
$$


Note that a minimizer of the variational problem

$$
\inf _{g, g(x)=1, x \in A}\left(E_{\nu_{N}^{\text {eq }}}\left[g^{2}\right]+\frac{\rho}{1-\rho} D_{\nu_{N}^{\text {eq }}}(g)\right)
$$

is the function $f$ defined above. We can choose $g=(1 / \exp (N l))\left(\exp \left(\sum_{i=1}^{N}\left|x_{i}\right|\right) \wedge \exp (N l)\right)$ and note that there exists a constant $C$ such that $E_{\nu_{N}^{\text {eq }}}\left[g^{2}\right] \leq C^{N}, D_{\nu_{N}^{\text {eq }}}(g) \leq C^{N}$. Therefore, we can bound $P_{N, T}^{\mathrm{eq}}(A)$ by $c^{N} \exp ^{-N l}$ and (3.19) is established.

One last comment, the tightness of the sequence $\left\{Q_{N, T}^{\mathrm{eq}}\right\}_{N}$ is valid without the assumption that the potential $V(x)$ is quadratic.

Energy estimate. Any limiting point $Q_{T}$ of the measure-valued sequence $\left\{Q_{N, T}^{\text {neq }}\right\}_{N}$ is supported on paths $\mu \in \mathscr{L}$ such that at each time $t, \mu(t)$ is a Lebesgue absolutely continuous measure on the torus $\mathbb{}$ with density $m(t, \theta)$. Moreover, for each finite time $T$, the density $m(t, \theta)$ satisfies the energy estimate

$$
\int_{0}^{T} \int_{\mathbb{T}}\left(\partial_{\theta}^{2} m(t, \theta)\right)^{2} d \theta d t<\infty
$$

We note that the energy estimate (3.23) is equivalent to the inequality

$$
\sup _{\phi \in C^{1,2}([0, T] \times \mathbb{T})} \int_{0}^{T} \int_{\mathbb{T}}\left(2 m(t, \theta) \partial_{\theta}^{2} \phi-C \phi^{2}\right) d \theta d t<\infty,
$$

where $C$ is a constant not depending on $\phi$.

The entropy inequality (3.5), the consequence of Feynman-Kac formula (4.18), and Lemma 4.2 can be used to derive the estimate

$$
\begin{aligned}
\limsup _{l, N \rightarrow \infty} \sup _{\phi \in C^{1,2}([0, T] \times \mathbb{T})} E^{\text {neq }} & {\left[\frac{1}{N} \sum_{i=1}^{N} \int_{0}^{T} 2 \phi\left(t, \frac{i}{N}\right) N^{2} \operatorname{Av}_{j=i-l+1}^{i+l-1}(\Delta x)_{j}\right.} \\
& \left.-\mathbb{V}_{i, l}(\Delta x, y) \phi^{2}\left(t, \frac{i}{N}\right) d t\right]<\infty .
\end{aligned}
$$

Here, the cylinder function $\Delta x$ is the discrete Laplacian of the slope field $x_{-1}-2 x_{0}+x_{1}$ and the variance $\mathbb{V}_{i, l}(\Delta x, y)$ is defined later in Section 4, see definition (4.44). As will be proved in Lemma 5.2, the variance $\mathbb{V}_{i, l}(\Delta x, y)$ has a uniform-in- $y$ lower bound, therefore the estimate (3.24) follows from (3.25), after integrating by parts. Moreover, we can conclude that at any time $t$, the weak second derivative of the measure $\mu(t)$ is in $L^{2}(\mathbb{T}, d \theta)$, and hence $\mu(t)$ is absolutely continuous with respect to Lebesgue measure on the torus $\mathbb{T}$.

Identification of the equation. That any limiting point of the measure-valued sequence $\left\{Q_{N, T}^{\text {neq }}\right\}_{N>0}$ is supported on the weak solutions of (2.15) follows if the event corresponding to the violation of the limiting equation has probability zero in the limit. 
For each test function $\phi \in C^{1,2}([0, T] \times \mathbb{T})$ that is differentiable in time and twice differentiable in space, and each finite time $T$, we define the function

$$
\begin{aligned}
V(t)= & \frac{1}{N} \sum_{i=1}^{N} \phi\left(t, \frac{i}{N}\right) x_{i}(t)-\frac{1}{N} \sum_{i=1}^{N} \phi\left(0, \frac{i}{N}\right) x_{i}(0) \\
& -\int_{0}^{t} \frac{1}{N} \sum_{i=1}^{N} \partial_{s} \phi\left(s, \frac{i}{N}\right) \bar{x}_{i, a N}(s) d s+\frac{1}{2} \int_{0}^{t} \frac{1}{N} \sum_{i=1}^{N} \phi^{\prime \prime}\left(s, \frac{i}{N}\right) \hat{a}\left(\bar{x}_{i, a N}(s)\right) \\
& \times b^{-2}\left(\bar{x}_{i-b N, c N}(s)-2 \bar{x}_{i, c N}(s)+\bar{x}_{i+b N, c N}(s)\right) d s
\end{aligned}
$$

and the event

$$
O_{a, b, c, \epsilon}^{\phi}=\left\{\sup _{0 \leq t \leq T}|V(t)|>\epsilon\right\} .
$$

We will prove in the next sections that for each $\epsilon>0$, we have

$$
\limsup _{a, b, c \rightarrow 0, N \rightarrow \infty} P_{N, T}^{\text {neq }}\left(O_{a, b, c, \epsilon}^{\phi}\right)=0 .
$$

The proof of the result (3.28) is complicated and is divided into several steps. The function that defines this event can be written as a sum of functions, see the beginning of Section 4. We will deal separately with each function in the sum and show that it converges to zero in probability.

\section{Identification of the limiting equation}

In this section, we establish that the event (3.27) is negligible in the limit, and hence any weak limit $Q_{T}$ is supported on the solutions of the Cauchy problem (2.15). To save space, we are suppressing the time dependence of the test function $\phi$ that defines the event (3.27).

Note on notations. Assume that $f$ is some local function. We denote by $\operatorname{Av}_{j=i-l}^{i+l} \tau^{j} f$ the average of shifts of $f$, namely,

$$
\frac{\tau^{i-l} f+\cdots+\tau^{i+l} f}{2 l+1}
$$

We write

$V(t)$

$$
\begin{aligned}
= & \frac{1}{N} \sum_{i=1}^{N} \phi\left(\frac{i}{N}\right) x_{i}(t)-\frac{1}{N} \sum_{i=1}^{N} \phi\left(\frac{i}{N}\right) x_{i}(0)-\int_{0}^{t} \frac{1}{N} \sum_{i=1}^{N} \phi\left(\frac{i}{N}\right) N^{4} L_{N}\left(x_{i}\right) d s \\
& +\frac{1}{2} \int_{0}^{t} \frac{1}{N} \sum_{i=1}^{N}\left\{N^{2}\left[\phi\left(\frac{i-1}{N}\right)-2 \phi\left(\frac{i}{N}\right)+\phi\left(\frac{i+1}{N}\right)\right]-\phi^{\prime \prime}\left(\frac{i}{N}\right)\right\} w_{i} d s
\end{aligned}
$$




$$
\begin{aligned}
& +\frac{1}{2} \int_{0}^{t} \frac{1}{N} \sum_{i=1}^{N} \phi^{\prime \prime}\left(\frac{i}{N}\right) N^{2}\left(w_{i}-\mathrm{Av}_{j=i-l_{1}}^{i+l_{1}} w_{j}\right) d s \\
& +\int_{0}^{t} \frac{1}{N} \sum_{i=1}^{N} \phi^{\prime \prime}\left(\frac{i}{N}\right) N^{2} \\
& \quad \times\left[\operatorname{Av}_{j=i-l_{1}}^{i+l_{1}} w_{j}-\hat{a}\left(\bar{x}_{i, l}\right) A_{j=i-l_{1}}^{i+l_{l_{1}}}(\Delta x)_{j}-\mathrm{Av}_{j=i-l_{1}}^{i+l_{1}} \tau^{j} L_{\infty} f_{r}\right] d s \\
& +\frac{1}{2} \int_{0}^{t} \frac{1}{N} \sum_{i=1}^{N} \phi^{\prime \prime}\left(\frac{i}{N}\right) N^{2} L_{\infty}\left(\operatorname{Av}_{j=i-l_{1}}^{i+l_{1}} \tau^{j} f_{r}\right) d s \\
& +\frac{1}{2} \int_{0}^{t} \frac{1}{N} \sum_{i=1}^{N} \phi^{\prime \prime}\left(\frac{i}{N}\right) N^{2} \\
& \quad \times\left[\hat{a}\left(\bar{x}_{i, l}\right) \operatorname{Av}_{j=i-l_{1}}^{i+l_{1}}(\Delta x)_{j}-\hat{a}\left(\bar{x}_{i, a N}\right) b^{-2}\left(\bar{x}_{i-b N, c N}-2 \bar{x}_{i, c N}+\bar{x}_{i+b N, c N}\right)\right] d s .
\end{aligned}
$$

We proceed to prove that each term in the sum above converges to 0 in probability.

Martingale estimate (the term (4.3)). We call $M_{N}(t)$ the term (4.3). From Itô formula, we know that the process $\left\{M_{N}(t)\right\}_{t \geq 0}$ is a martingale and

$$
E^{\mathrm{neq}}\left[M_{N}^{2}(T)\right]=E^{\mathrm{neq}}\left[\int_{0}^{T} \frac{1}{N^{2}} \sum_{i=1}^{N} N^{4}\left[\phi\left(\frac{i-1}{N}\right)-2 \phi\left(\frac{i}{N}\right)+\phi\left(\frac{i+1}{N}\right)\right]^{2} a_{i} d s\right] .
$$

The test function $\phi$ is chosen to have continuous second derivatives, then $E^{\text {neq }}\left[M_{N}^{2}(T)\right]$ is of order $O(1 / N)$. We can use Doob's inequality

$$
P_{N, T}^{\text {neq }}\left\{\sup _{0 \leq t \leq T}\left|M_{N}(t)\right| \geq \epsilon\right\} \leq \frac{1}{\epsilon^{2}} E\left[M_{N}^{2}(T)\right], \quad \epsilon>0,
$$

to conclude that the martingale is negligible in the limit, that is,

$$
\lim _{N \rightarrow \infty} P_{N, T}^{\text {neq }}\left\{\sup _{0 \leq t \leq T}\left|M_{N}(t)\right| \geq \epsilon\right\}=0, \quad \epsilon>0 .
$$

The term (4.4). A straightforward computation involving the Chebyshev inequality and the entropy inequality (3.5) proves that the term (4.4) converges in probability to 0 . The test function needs to have continuous fourth derivative.

A technical lemma. We will prove a lemma that reduces the problem of establishing the negligibility of an event to finding that the largest eigenvalue of a Schrödinger operator is negative. For an operator $A: \mathcal{H} \rightarrow \mathcal{H}$ acting on a Hilbert space $\mathscr{H}$, we denote by $\operatorname{supsec}_{\mathscr{H}} A$ the largest value in the spectrum of $A$. 
LEMmA 4.1. Let $\{x(t)\}_{t \geq 0}$ be the slope process with generator (2.6). Under the assumption

$$
\begin{aligned}
& \underset{N \rightarrow \infty}{\limsup } \underset{L^{2}\left(\nu_{N}^{\text {eq }}\right)}{\operatorname{supspec}}\left(\alpha g+\frac{N^{4}}{N} L_{N}\right) \\
& \quad=\limsup _{N \rightarrow \infty} \sup _{\rho, E^{\text {eq }}\left[\rho^{2}\right]=1}\left[\alpha E^{\mathrm{eq}}\left[g \rho^{2}\right]-\frac{N^{4}}{N} D_{N}(\rho)\right] \leq 0, \quad \alpha \neq 0,
\end{aligned}
$$

it follows that the event $\left\{\left|\int_{0}^{T} g(x(s)) d s\right| \geq \epsilon\right\}$ has negligible probability or

$$
\lim _{N \rightarrow \infty} P_{N, T}^{\text {neq }}\left\{\left|\int_{0}^{T} g(x(t)) d t\right| \geq \epsilon\right\}=0, \quad \epsilon>0 .
$$

Proof. We can use the Chebyshev inequality to reduce the proof of (4.13) to

$$
\lim _{N \rightarrow \infty} E^{\text {neq }}\left[\left|\int_{0}^{T} g(x(t)) d t\right|\right]=0
$$

Since we do not have much information about the initial nonequilibrium distribution, we use the entropy inequality to replace the nonequilibrium distribution in (4.14) by the equilibrium distribution.

In our context, we use the entropy inequality (3.5) for the distribution of the process started in the nonequilibrium and the distribution of the process started in the equilibrium. We have

$$
\begin{aligned}
E^{\text {neq }}[\mid & \left.\int_{0}^{T} g(x(t)) d t \mid\right] \\
& =E^{\text {neq }}\left[\frac{1}{N \alpha}\left|\int_{0}^{T} N \alpha g(x(t)) d t\right|\right] \\
& \leq \frac{1}{N \alpha} H\left(\nu_{N}^{\mathrm{neq}} \mid \nu_{N}^{\mathrm{eq}}\right)+\frac{1}{N \alpha} \log E^{\mathrm{eq}}\left[\exp \left(\alpha\left|\int_{0}^{T} N g(x(t)) d t\right|\right)\right] \\
& \leq \frac{C}{\alpha}+\frac{1}{N \alpha} \log E^{\mathrm{eq}}\left[\exp \left(N \alpha \int_{0}^{T} g(x(t)) d t\right)+\exp \left(-N \alpha \int_{0}^{T} g(x(t)) d t\right)\right] .
\end{aligned}
$$

As a consequence of the inequality

$$
\log (a+b) \leq \max (\log (2 a), \log (2 b)) \leq \log (2)+\max (\log (a), \log (b))
$$

for two positive numbers $a$ and $b,(4.14)$ follows as soon as we have

$$
\underset{N \rightarrow \infty}{\limsup } \frac{1}{N} \log E^{\mathrm{eq}}\left[\exp \left(N \alpha \int_{0}^{T} g(x(t)) d t\right)\right] \leq 0, \quad \alpha \neq 0
$$


A trivial consequence of Feynman-Kac formula proves our lemma,

$$
\frac{1}{N} \log E^{\mathrm{eq}}\left[\exp \left(N \alpha \int_{0}^{T} g(x(t)) d t\right)\right] \leq T \underset{L^{2}\left(\nu_{N}^{\mathrm{eq}}\right)}{\operatorname{supspec}}\left(\alpha g+\frac{N^{4}}{N} L_{N}\right) .
$$

The microscopic current $w$ can be replaced by local average of currents (the term (4.5)). We will show that the term (4.5) converges to zero in probability. As a consequence the current $w$ is replaced by a local average of $w$ that is closer to a deterministic value. $w$ by itself is a single fluctuating random variable.

We check that the hypothesis of Lemma 4.1 is valid for the function $g_{l, N}=(1 /$ N) $\sum_{i=1}^{N} \phi(i / N) N^{2}\left(w_{i}-\mathrm{Av}_{j=i-l}^{i+l} w_{j}\right)$. Recall that if the test function $\phi$ has continuous second-order derivative, the quantity

$$
\operatorname{Av}_{j=i-l}^{i+l} \phi\left(\frac{j}{N}\right)-\phi\left(\frac{i}{N}\right)=\frac{\phi((i-l) / N)-\phi(i / N)+\cdots+\phi((i+l) / N)-\phi(i / N)}{2 l+1}
$$

is of order $O\left(l^{2} / N^{2}\right)$. Now, on integrating by parts twice, it follows for a fixed function $\rho$, with $E^{\mathrm{eq}}\left[\rho^{2}\right]=1$, that

$$
\begin{aligned}
\mid E^{\mathrm{eq}}[ & \left.\rho^{2} \frac{1}{N} \sum_{i=1}^{N} \phi\left(\frac{i}{N}\right) N^{2}\left(w_{i}-\mathrm{Av}_{j=i-l}^{i+l} w_{j}\right)\right]\left.\right|^{2} \\
& =4\left|E^{\mathrm{eq}}\left[\frac{N^{2}}{N} \sum_{i=1}^{N} \sqrt{a_{i}} X_{i}(\rho) \sqrt{a_{i}}\left(A v_{j=i-l}^{i+l} \phi\left(\frac{j}{N}\right)-\phi\left(\frac{i}{N}\right)\right) \rho\right]\right|^{2} \\
& \leq 4 E^{\mathrm{eq}}\left[\sum_{i=1}^{N} \frac{N^{4}}{N^{2}} a_{i}\left(\mathrm{Av}_{j=i-l}^{i+l} \phi\left(\frac{j}{N}\right)-\phi\left(\frac{i}{N}\right)\right)^{2} \rho^{2}\right] D_{N}(\rho) \leq C \frac{l^{4}}{N^{4}} \frac{N^{4}}{N} D_{N}(\rho) .
\end{aligned}
$$

Therefore,

$$
\limsup _{l, N \rightarrow \infty} \sup _{\rho, E^{\mathrm{eq}}\left[\rho^{2}\right]=1} \frac{\left(E^{\mathrm{eq}}\left[\rho^{2} N \sum_{i=1}^{N}\left(\mathrm{Av}_{j=i-l}^{i+l} \phi(j / N)-\phi(i / N)\right) w_{i}\right]\right)^{2}}{D_{N}(\rho)} \frac{N}{N^{4}}=0
$$

and hence (4.12) is satisfied. Moreover,

$$
\lim _{l, N \rightarrow \infty} P_{N, T}^{\mathrm{neq}}\left\{\left|\int_{0}^{T} \frac{1}{N} \sum_{i=1}^{N} \phi\left(\frac{i}{N}\right) N^{2}\left(w_{i}-\mathrm{Av}_{j=i-l}^{i+l} w_{j}\right) d t\right| \geq \epsilon\right\}=0, \quad \epsilon>0 .
$$

Inserting the fluctuations (the term (4.7)). Let $f\left(x_{-s}, \ldots, x_{s}, \bar{x}_{0, l}\right) \in C^{2}\left(\mathbb{R}^{2 s+1}\right)$ be a local function that depends on the slope configuration in a box of size $s$ and on the mean slope in a box of a large size $l$. We use the notation $l_{1}=l-\sqrt{l}$ for a slightly smaller $l$. We want 
to show that the fluctuations approach zero in the limit or that

$$
\lim _{l, N \rightarrow \infty} P_{N, T}^{\text {neq }}\left\{\left|\int_{0}^{T} \frac{N^{2}}{N} \sum_{i=1}^{N} \phi\left(\frac{i}{N}\right) L_{N}\left(\operatorname{Av}_{j=i-l_{1}}^{i+l_{1}} \tau^{j} f\right) d t\right| \geq \epsilon\right\}=0, \quad \epsilon>0 .
$$

We apply Itô formula,

$$
\begin{aligned}
& \int_{0}^{T} \frac{N^{2}}{N} \sum_{i=1}^{N} \phi\left(\frac{i}{N}\right) L_{N}\left(A v_{j=i-l_{1}}^{i+l_{l_{1}}} \tau^{j} f\right) d t \\
& \quad=\frac{1}{N \cdot N^{2}} \sum_{i=1}^{N} \phi\left(\frac{i}{N}\right)\left[\left(A v_{j=i-l_{1}}^{i+l_{1}} \tau^{j} f\right)(x(T))-\left(A v_{j=i-l_{1}}^{i+l_{1}} \tau^{j} f\right)(x(0))\right]+\frac{1}{N^{2}} M_{N}(t) .
\end{aligned}
$$

The first summand in (4.24) converges to zero, as the function $f$ is bounded. The second part of (4.24) approaches zero because the $L^{2}$ norm of $M_{N}(T) / N^{2}$ is of order $\mathcal{O}\left(l^{2} / N\right)$, as we can see below. Let $g=(1 / N) \sum_{i=1}^{N} \phi\left(l^{2} / N\right)\left(\mathrm{Av}_{j=i-l_{1}}^{i+l_{1}} \tau^{j} f\right)(x)$, then

$$
\begin{aligned}
E^{\mathrm{neq}}\left[\frac{M_{N}(T)^{2}}{N^{4}}\right] & =E^{\mathrm{neq}}\left[\int_{0}^{T} L_{N} g-2 g L_{N} g d t\right]=E^{\mathrm{neq}}\left[\int_{0}^{T} \sum_{i=1}^{N} a_{i}\left(X_{i} g\right)^{2} d t\right] \\
& =\frac{1}{N^{2}} E^{\mathrm{neq}}\left[\int_{0}^{T} \sum_{i=1}^{N} a_{i}\left(\sum_{k=1}^{N} \phi\left(\frac{k}{N}\right) X_{i}\left(\mathrm{Av}_{j=k-l_{1}}^{k+l_{1}} \tau^{j} f\right)\right)^{2} d t\right] .
\end{aligned}
$$

The function $\mathrm{Av}_{j=k-l_{1}}^{k+l_{1}} \tau^{j} f$ is a cylinder function that depends just on the sites $n$ such that $k-l \leq n \leq k+l$. Therefore the vector field $X_{i}$ is zero when acting on most of the summands inside $A v_{j=k-l_{1}}^{k+l_{1}} \tau^{j} f$. There are no more than $2 l$ sites $k$ such that $X_{i}\left(\operatorname{Av}_{j=k-l_{1}}^{k+l_{1}} \tau^{j} f\right) \neq$ 0 . We put all these arguments together to conclude that $E^{\text {neq }}\left[M^{2}(t) / N^{4}\right] \leq C l^{2} / N$.

Replacing the current by the Laplacian of the slope field (the term (4.6)). In our model, the instantaneous current $w$ cannot be written as the discrete Laplacian $\tau h-2 h-\tau^{-1} h$ of some local function $h$, thus we use the method of Varadhan [19] for computing the hydrodynamic scaling limit of our model. The main idea is that the current decomposes as

$$
w=\hat{a}\left(\bar{x}_{l}\right) \Delta x+L_{\infty} f
$$

for a suitable coefficient $\hat{a}\left(\bar{x}_{l}\right)$, where $\bar{x}_{l}$ is the average slope in a cube centered at the origin of microscopic site $l$. Equation (4.26) is known in the literature as the fluctuationdissipation equations. We have explained before that terms of the form $L_{\infty} f$ have no effect on the macroscopic scale.

A new feature is characteristic to our model due to the complex interaction of the system: after filtering off the fluctuations from the current, we are left with the Laplacian of some function and not a gradient, as happened for models previously considered in the literature. The precise meaning of (4.26) is given below. 
Our aim is to prove the existence of a sequence $\left\{f_{r}\right\}_{r \geq 0}$ of local functions and of the transport coefficient $\hat{a}$ such that

$$
\begin{array}{r}
\limsup _{r, l, N \rightarrow \infty} P_{N, T}^{\text {neq }}\left\{\mid \int_{0}^{T} \frac{N^{2}}{N} \sum_{i=1}^{N} \phi\left(\frac{i}{N}\right)\left[A v_{j=i-l_{1}}^{i+l_{1}} w_{j}-\hat{a}\left(\bar{x}_{i, l}\right) \operatorname{Av}_{j=i-l_{1}}^{i+l_{1}}(\Delta x)_{j}\right.\right. \\
\left.\left.-\mathrm{Av}_{j=i-l_{1}}^{i+l_{1}} \tau^{j} L_{\infty} f_{r}\right] d t \mid \geq \epsilon\right\}=0 .
\end{array}
$$

The local function $f_{r}\left(x_{-s}, \ldots, x_{s}, \bar{x}_{0, l}\right)$ depends on two arguments, the mean slope $\bar{x}_{0, l}$ in a box of size $l$ and the slope field inside a box of size $s$, the size $s$ being much smaller than $l$. We can assume that the operator $L_{\infty}$ does not act on the first argument $\bar{x}_{0, l}$, since we can show that the action of the operator $L_{\infty}$ at the boundary sites is negligible. To be more precise, $\mathrm{Av}_{j=i-l_{1}}^{i+l_{1}} \tau^{j} L_{\infty} f_{r}$ stands for

$$
\frac{L_{\infty} f_{r}\left(\bar{x}_{i, l}, \tau_{i-l_{1}} x\right)+\cdots+L_{\infty} f_{r}\left(\bar{x}_{i, l}, \tau_{i+l_{1}} x\right)}{2 l_{1}+1} .
$$

Note that the function $A v_{j=i-l_{1}}^{i+l_{1}} \tau^{j} L_{\infty} f_{r}$ depends just on the value of the field inside the box centered at $i$ and of size $l$.

As before, we use Lemma 4.1 to conclude that the event (4.27) has negligible probability in the limit. An additional difficulty shows up. If $\lambda_{\epsilon}$ is the largest eigenvalue of the perturbation $L+\epsilon W$ of a negative operator $L$ with principal eigenvalue 0 , the eigenvalue $\lambda_{\epsilon}$ has the formal series expansion,

$$
\lambda_{\epsilon}=0+\epsilon E_{\nu}[W]+\epsilon^{2}\left\langle W,(-L)^{-1} W\right\rangle_{\nu}+O\left(\epsilon^{3}\right) .
$$

Hence if the potential $W$ has mean zero, one expects $\lim _{\epsilon \rightarrow 0} \lambda_{\epsilon} \epsilon^{-2}=\left\langle W,(-L)^{-1} W\right\rangle_{\nu}$. Fortunately for suitable potential $W$, the central limit variance $\left\langle W,(-L)^{-1} W\right\rangle_{\nu}$ converges to zero.

We will need in our context a particular result about the largest eigenvalue of a perturbation operator. The reader can find further details in Quastel in [15].

LeMma 4.2. Let $W$ be a real potential that satisfies

$$
\langle u, W u\rangle_{v} \leq l^{-1 / 2} D_{l}(u)^{1 / 2}\|u\|_{2}, \quad l<C \epsilon^{-2 / 5}
$$

for some C small enough, or

$$
\|W\|_{\infty} \leq C, \quad K \leq(C \epsilon)^{-1 / 5} .
$$

Provided that the generator $l^{4} L_{l}$ has spectral gap of order one, the following estimate holds:

$$
\epsilon^{-2} l^{-5} \operatorname{supspec}_{L^{2}(v)}\left(l^{4} L_{l}-\epsilon l^{5} W\right) \leq l\left\langle W,\left(-L_{l}\right)^{-1} W\right\rangle_{\nu}+O(1) .
$$


Spectral gap. Indeed the generator $l^{4} L_{l}$ of our model, defined by (2.9), has a spectral gap of order 1. The proof is standard by the Bakry-Emery method (see Chang and Yau [3] or Deuschel and Stroock [4]).

The operator $L_{l}$ is an unbounded operator defined on the subspace $C_{0}^{\infty}\left(\mathbb{R}^{2 l+1}\right)$ of the Hilbert space $L^{2}\left(v_{\alpha, l}^{\mathrm{gc}}\right)$ and is negative definite, with spectrum included in the negative semiaxis of the real line. Zero is an eigenvalue of the operator $L_{l}$ but the eigenspace corresponding to this eigenvalue is quite large, being infinite dimensional.

It is not hard to see that we can write the Hilbert space $L^{2}\left(v_{\alpha, l}^{\mathrm{gc}}\right)$ as the direct sum $\bigoplus_{y \in \mathbb{R}^{2}} L^{2}\left(v_{y, l}^{c}\right)$. Moreover because of the ergodicity of the dynamics (2.9) on the level sets of the function $y_{0, l}=\left(y_{0, l}^{1}, y_{0, l}^{2}\right)$, we know that the eigenspace corresponding to zero of the restriction of the operator $L_{l}$ onto each Hilbert subspace $L^{2}\left(v_{y, l}^{c}\right)$ is one dimensional. The next eigenvalue of the restriction $\left.L_{l}\right|_{L^{2}\left(v_{y, l}^{c}\right)}$ is a negative number. The distance between the largest eigenvalue and the next largest eigenvalue of the operator $\left.L_{l}\right|_{L^{2}\left(v_{y, l}^{c}\right)}$ is called the spectral gap of the operator, because it is the gap in the spectrum of the operator.

We provide next a bound on the size of the spectral gap of an operator more general than $L_{l}$.

Lemma 4.3. Suppose that $L$ is the unique symmetric operator given by the quadratic form

$$
D_{L}(\rho)=\langle(-L) \rho, \rho\rangle_{\nu}=\int_{\mathbb{R}^{N}} \sum_{i=1}^{N}\left(\partial_{i} \rho\right)^{2} e^{-U(x)} d x .
$$

The function $U$ is such that at each point $x \in \mathbb{R}^{N}$, the Hessian of $U$, $(\operatorname{Hess} U)(x)=$ $\left\{\partial_{i} \partial_{j}(U(x))\right\}_{1 \leq i, j \leq N}$, has the property $\langle(\operatorname{Hess} U)(x) v, v\rangle \geq C\|v\|^{2}, v \in \mathbb{R}^{N}$. The constant $C$ is supposed to be independent of the point $x$. Under these assumptions, L has a gap in the spectrum of size at least $C$ and that for any $\rho$ such that $E_{\nu}[\rho]=0$,

$$
C E_{\nu}\left[\rho^{2}\right] \leq\langle(-L) \rho, \rho\rangle_{\nu} .
$$

Proof. Integrating by parts in (4.33), we find the form of the operator $-L$ :

$$
(-L) \rho=\sum_{i=1}^{N}\left[-\partial_{i}^{2}(\rho)+\partial_{i}(U) \partial_{i}(\rho)\right]
$$

Assume that $\lambda$ is a nonzero eigenvalue of $-L$ and $\rho$, with the property that $E_{\gamma}[\rho]$ is the corresponding eigenfunction such that $(-L) \rho=\lambda \rho$. In both sides of this equality, we differentiate with respect to the $j^{\text {th }}$ coordinate and multiply with $\partial_{j}(\rho)$ to get

$$
\sum_{i=1}^{N}\left[-\partial_{j} \partial_{i}^{2}(\rho) \partial_{j}(\rho)+\partial_{j} \partial_{i}(U) \partial_{i}(\rho) \partial_{j}(\rho)+\partial_{i}(U) \partial_{j} \partial_{i}(\rho) \partial_{j}(\rho)\right]=\lambda\left(\partial_{j}(\rho)\right)^{2} .
$$

We sum over all possible values of $j$ and then integrate over $\mathbb{R}^{N}$ in (4.36) to get

$$
\int_{\mathbb{R}^{N}} \sum_{1 \leq i, j \leq N}\left[\left(\partial_{i} \partial_{j}(\rho)\right)^{2}+\left(\partial_{i} \partial_{j}\right)(U) \partial_{i}(\rho) \partial_{j}(\rho)\right] d \nu=\lambda \int_{\mathbb{R}^{N}} \sum_{i=1}^{N}\left(\partial_{i}(\rho)\right)^{2}(x) d \nu .
$$

Since $\rho$ is not a constant function, it follows immediately that the eigenvalue $\lambda \geq C$. 
Lemma 4.4 (spectral gap). There is a universal constant $C$ that does not depend on the conserved quantities $y_{0, l}=\left(y_{0, l}^{1}, y_{0, l}^{2}\right) \in \mathbb{R}^{2}$ such that

$$
\frac{C}{l^{4}} E_{v_{y, l}^{c}}\left[\rho^{2}\right] \leq\left\langle\left(-L_{l}\right) \rho, \rho\right\rangle_{v_{y, l}^{c}}
$$

for any mean-zero function $\rho, E_{\gamma_{y, l}^{c}}[\rho]=0$.

Proof. To save on notations, throughout the proof of this lemma, we label the coordinates of the underlying space $\mathbb{R}^{2 l+1}$ of the measure $v_{y, l}^{c}$ by $x_{0}, x_{1}, \ldots, x_{2 l}$.

First, we observe that the canonical measure $v_{y, l}^{c}$ satisfies the hypothesis of Lemma 4.3. For this, we need a formula for the expectation of a function $\rho$ with respect to the canonical measure. Any point $x \in \mathbb{R}^{2 l+1}$ on the level set $\left(y_{0, l}^{1}=x_{0}+\cdots+x_{2 l}, y_{0, l}^{2}=x_{1}+2 x_{2}+\right.$ $\left.\cdots+2 l x_{2 l}\right)$ can be thought of as being a point in the image of the function

$$
f\left(x_{2}, \ldots, x_{2 l}\right)=\left(y_{0, l}^{1}-y_{0, l}^{2}+x_{2}+\cdots+(2 l-1) x_{2 l}, y_{0, l}^{2}-2 x^{2}-\cdots-2 l x_{2 l}, x_{2}, \ldots, x_{2 l}\right) .
$$

This allows us to write

$$
E_{\gamma_{y, l}^{c}}[\rho]=\frac{\int_{\mathbb{R}^{2 l-1}} \rho\left(f\left(x_{2}, \ldots, x_{2 l}\right)\right) e^{-U\left(x_{2}, \ldots, x_{2 l}\right)} d x_{2} \cdots d x_{2 l}}{\int_{\mathbb{R}^{2 l-1}} e^{-U\left(x_{2}, \ldots, x_{2 l}\right)} d x_{2} \cdots d x_{2 l}},
$$

where $U\left(x_{2}, \ldots, x_{2 l+1}\right)=H_{2 l+1}(f(x))$.

We calculate the Hessian of $U$ to get

$$
\begin{aligned}
\operatorname{Hess}(U)= & V^{\prime \prime}\left(y_{0, l}^{1}-y_{0, l}^{2}+x_{2}+\cdots+(2 l-1) x_{2 l}\right) M_{1} \\
& +V^{\prime \prime}\left(y_{0, l}^{2}-2 x^{2}-\cdots-2 l x_{2 l}\right) M_{2}+D,
\end{aligned}
$$

where $M_{1}=\{(i-1)(j-1)\}_{2 \leq i, j \leq 2 l}, M_{2}=\{i j\}_{2 \leq i, j \leq 2 l}$ are two positive matrices and $D$ is a diagonal matrix such that the entries on the main diagonal are $D(i, i)=V^{\prime \prime}\left(x_{i}\right), 2 \leq i \leq 2 l$. If $V^{\prime \prime}$ is uniformly bounded from below by some constant $C$, then we obtain the required condition $\langle\operatorname{Hess}(U) v, v\rangle \geq C\|v\|^{2}$ for all vectors $v \in \mathbb{R}^{2 l-1}$. Note that for a quadratic potential $V(x)=x^{2} / 2$, this condition is satisfied, $V^{\prime \prime}(x)=1$.

Recall that the vector field $X_{i}=\partial_{i-1}-2 \partial_{i}+\partial_{i+1}$. Before proving this result, we must note that for a smooth function $\rho$, we have

$$
\partial_{i}(\rho \circ f)=\left[\left((i-1) \partial_{0}(\rho)-i \partial_{1}(\rho)+\partial_{i}\right) \rho\right] \circ f=\left[\left(X_{i-1}+2 X_{i-2}+\cdots+(i-1) X_{1}\right) \rho\right] \circ f .
$$

It follows from Lemma 4.3 that there is a constant independent of $y_{0, l}=\left(y_{0, l}^{1}, y_{0, l}^{2}\right)$ such that

$$
C E_{\gamma_{y, l}^{c}}\left[\rho^{2}\right] \leq E_{\gamma_{y, l}^{c}}\left[\sum_{i=2}^{2 l}\left[\left(X_{i-1}+2 X_{i-2}+\cdots+(i-1) X_{1}\right) \rho\right]^{2}\right] \leq l^{4} E_{\gamma_{y, l}^{c}}\left[\sum_{i=1}^{2 l-1}\left(X_{i}(\rho)\right)^{2}\right] .
$$

Therefore, the operator $-\left.L_{l}\right|_{L^{2}\left(v_{y, l}^{c}\right)}$ has a gap in the spectrum of order $1 / l^{4}$. 
Since the generator $L_{l}$ of our model has a spectral gap, see Lemma 4.4, we can introduce the central limit theorem variance in our context.

Definition 4.5. Let $f\left(x_{-s}, \ldots, x_{s}\right)$ be a cylinder function such that $E_{\gamma_{y, s}^{c}}[f]=0$ for all possible values of $y \in \mathbb{R}^{2}$. Recall that $\nu_{y, s}^{c}$ is the canonical measure in a box centered at 0 and size $s$ defined in Section 2. The central limit theorem variance (CLT-variance) of $f$ on the box $\Lambda_{i, l}$ is defined to be

$$
\mathbb{V}_{i, l}(f, y)=2(2 l)\left\langle A v_{j=i-l_{1}}^{i+l_{1}} \tau^{j} f,\left(-L_{i, l}\right)^{-1}\left(\operatorname{Av}_{j=i-l_{1}}^{i+l_{l}} \tau^{j} f\right)\right\rangle_{\gamma_{y, i, l}^{c}}
$$

If the box $\Lambda_{i, l}$ is centered at 0 , then use the shorter notation $\mathbb{V}_{l}(f, y)$ for the CLT-variance.

At this point, we stress that $\mathbb{V}_{i, l}(f, y)$ is a local function depending on the field inside $\Lambda_{i, l}$, more precisely depending on the conserved quantities $y_{i, l}=\left(y_{i, l}^{1}, y_{i, l}^{2}\right)$, the mean slope, and the linear mean of the slope field.

The strategy is to give a bound for the largest eigenvalue of a perturbation operator in terms of the CLT-variance $\mathbb{V}_{i, l}(f, y)$. Extra care must be taken because the CLT-variance $\mathbb{V}_{i, l}(f, y)$ is uniformly-in- $y$ small on bounded sets and not on unbounded sets.

The canonical measure $v_{i, l, y}^{c}$ for our model has been obtained by conditioning the grand canonical measure on the configurations with fixed mean slope and fixed linear mean slope in a box centered at $i$ and of size $l$. The second conditioning makes the canonical measure not having identical marginals. Actually, the expected values of the marginals depend linearly on the site. However the finite-dimensional marginals of the canonical measure converge towards the finite-dimensional marginals of the grand canonical distribution as the size of the box approaches infinity, see Lemma 5.1. To benefit from this fact, we will replace the CLT-variance $\mathbb{V}_{l}(f, y)$ in a box of size $l$ with its expectation $E^{\mathrm{eq}}\left[\mathbb{V}_{l}(f, y) \mid y_{k}\right]$ with respect to the canonical measure $v_{y, k}^{c}$ in a box of larger size $k$. We let $k$ go first to infinity. We formalize below.

Let us define the function $g$ as

$$
g=\frac{N^{2}}{N} \sum_{i=1}^{N} \phi\left(\frac{i}{N}\right)\left[\mathrm{Av}_{j=i-l_{1}}^{i+l_{1}} w_{j}-\hat{a}\left(\bar{x}_{i, l}\right) \mathrm{Av}_{j=i-l_{1}}^{i+l_{1}}(\Delta x)_{j}-\mathrm{Av}_{j=i-l_{1}}^{i+l_{1}} \tau^{j} L_{\infty} f_{r}\right]
$$

Thanks to Lemma 4.1, the event (4.27) has negligible probability if

$$
\limsup _{r, l, N \rightarrow \infty} \operatorname{supspec}_{L^{2}\left(\nu_{N}^{\text {eq }}\right)}\left(g+2 \beta \frac{N^{4}}{N} L_{N}\right) \leq 0, \quad \beta>0 .
$$

We write the operator $g+2 \beta\left(N^{4} / N\right) L_{N}$ as a sum of operators and we estimate the size of the principal eigenvalue of each operator in the sum

$$
g+2 \beta \frac{N^{4}}{N} L_{N}=\Omega_{1}+\Omega_{2}+\Omega_{3}+\Omega_{4}
$$


where

$$
\begin{aligned}
& \Omega_{1}=g-\frac{1}{\beta N} \sum_{i=1}^{N} \phi\left(\frac{i}{N}\right)^{2} \mathbb{V}_{i, l}\left(w-\hat{a}\left(\bar{x}_{i, l}\right) \Delta x-L_{\infty} f_{r}, y\right)+\beta \frac{N^{4}}{N} L_{N}, \\
& \Omega_{2}=\frac{1}{\beta N} \sum_{i=1}^{N} \phi\left(\frac{i}{N}\right)^{2}\left[\mathbb{V}_{i, l}\left(w-\hat{a}\left(\bar{x}_{i, l}\right) \Delta x-L_{\infty} f_{r}, y\right)\right. \\
& \left.\qquad-E^{\mathrm{eq}}\left[\mathbb{V}_{i, l}\left(w-\hat{a}\left(\bar{x}_{i, l}\right) \Delta x-L_{\infty} f_{r}, y\right) \mid y_{i, k}\right]\right]+\beta \frac{N^{4}}{N} L_{N}, \\
& \Omega_{3}=\frac{1}{\beta N} \sum_{i=1}^{N} \phi\left(\frac{i}{N}\right)^{2} E^{\mathrm{eq}}\left[\mathbb{V}_{i, l}\left(w-\hat{a}\left(\bar{x}_{i, l}\right) \Delta x-l_{\infty} f_{r}, y\right) \mid y_{i, k}\right] \mathbf{1}_{\left|y_{i, k}\right| \geq \delta}, \\
& \Omega_{4}=\frac{1}{\beta N} \sum_{i=1}^{N} \phi\left(\frac{i}{N}\right)^{2} E^{\mathrm{eq}}\left[\mathbb{V}_{i, l}\left(w-\hat{a}\left(\bar{x}_{i, l}\right) \Delta x-L_{\infty} f_{r}, y\right) \mid y_{i, k}\right] \mathbf{1}_{\left|y_{i, k}\right| \leq \delta .}
\end{aligned}
$$

The operators $\Omega_{2}, \Omega_{3}$, and $\Omega_{4}$ are understood as multiplication operators.

The operator $\Omega_{1}$. Assume that $M$ is an upper bound for the test function $|\phi|$. Define

$$
g_{i, l}=\operatorname{Av}_{j=i-l_{1}}^{i+l_{1}} w_{j}-\hat{a}\left(\bar{x}_{i, l}\right) \operatorname{Av}_{j=i-l_{1}}^{i+l_{1}}(\Delta x)_{j}-\operatorname{Av}_{j=i-l_{1}}^{i+l_{1}} \tau^{j} L_{\infty} f_{r}
$$

We have

$$
\begin{aligned}
& \operatorname{supspec}_{L^{2}\left(\nu_{N}^{\text {eq }}\right)}\left(\Omega_{1}\right) \leq \sup _{|\lambda| \leq M} \sup _{\rho, E^{\text {eq }}\left[\rho^{2}\right]=1}\left[E^{\text {eq }}\left[\lambda N^{2} g_{0, l} \rho^{2}-\frac{\lambda^{2}}{\beta} \mathbb{V}_{l}\left(w-\hat{a}\left(\bar{x}_{l}\right) \Delta x-L_{\infty} f_{r}, y\right) \rho^{2}\right]\right. \\
& \left.-\frac{\beta N^{4}}{2 l+1} D_{l}(\rho)\right] \\
& \leq \sup _{|\lambda| \leq M} \sup _{y_{l} \in \mathbb{R}^{2}}\left[\sup _{\rho, E^{\mathrm{eq}}\left[\rho^{2} \mid y_{l}\right]=1}\left[E^{\mathrm{eq}}\left[\lambda N^{2} g_{0, l} \rho^{2}\right]-\frac{\beta N^{4}}{2 l+1} D_{\gamma_{y, l}^{c}}(\rho)\right]\right. \\
& \left.-\frac{\lambda^{2}}{\beta} \mathbb{V}_{l}\left(w-\hat{a}\left(\bar{x}_{l}\right) \Delta x-L_{\infty} f_{r}, y\right)\right] \text {. }
\end{aligned}
$$

Integrating by parts, we can show that there is a constant $C$, not depending on $l$ and the values of the conserved quantities, such that for each density $\rho$,

$$
E^{\mathrm{eq}}\left[g_{0, l} \rho^{2} \mid y_{l}\right] \leq C \frac{\sqrt{D_{v_{y, l}^{c}}(\rho)}}{\sqrt{2 l+1}} .
$$


Hence, the hypothesis of Lemma 4.2 is satisfied and

$$
\begin{aligned}
& \limsup _{N \rightarrow \infty} \sup _{\rho, E^{\mathrm{eq}}\left[\rho^{2} \mid y_{l}\right]=1}\left[E^{\mathrm{eq}}\left[\lambda N^{2} g_{0, l} \rho^{2}\right]-\frac{\beta N^{4}}{2 l+1} D_{\gamma_{y, l}^{c}}\left(\rho^{2}\right)\right] \\
& \quad=\frac{1}{(2 l+1) l^{4}} \limsup _{N \rightarrow \infty} N^{4} \sup _{\rho, E^{\mathrm{eq}}\left[\rho^{2} \mid y_{l}\right]=1}\left[E^{\mathrm{eq}}\left[\frac{\lambda(2 l+1) l^{4}}{N^{2}} g_{0, l} \rho^{2}\right]-\beta l^{4} D_{\gamma_{y, l}^{c}}\left(\rho^{2}\right)\right] \\
& \quad \leq \frac{\lambda^{2}}{\beta} \mathbb{V}_{l}\left(w-\hat{a}\left(\bar{x}_{l}\right) \Delta x-L_{\infty} f_{r}, y\right) .
\end{aligned}
$$

The convergence on the line above is uniform over the set of all possible values of the conserved quantity $y_{l}=\left(y_{l}^{1}, y_{l}^{2}\right) \in \mathbb{R}^{2}$, therefore the principal eigenvalue of the operator $\Omega_{1}$ becomes negative as $N \rightarrow \infty$.

The operator $\Omega_{2}$. Let us define

$$
v_{i, k}=V_{i, l}\left(w-\hat{a}\left(\bar{x}_{i, l}\right) \Delta x-L_{\infty} f_{r}, y\right)-E^{e q}\left[V_{i, l}\left(w-\hat{a}\left(\bar{x}_{i, l}\right) \Delta x-L_{\infty} f_{r}, y\right) \mid y_{i, k}\right] .
$$

We observe that $\Omega_{2}$ contains $v_{i, k}$ without being multiplied with a factor $N^{2}$. Then,

$$
\begin{aligned}
& \underset{L^{2}\left(\nu_{N}^{\mathrm{eq}}\right)}{\operatorname{suppec}}\left(\Omega_{2}\right)=\underset{L^{2}\left(\nu_{N}^{\mathrm{eq}}\right)}{\operatorname{supspec}}\left[\frac{1}{\beta N} \sum_{i} \phi\left(\frac{i}{N}\right)^{2} v_{i, k}+\beta \frac{N^{4}}{N} L_{N}\right] \\
& \leq \sup _{|\lambda| \leq M} \sup _{y_{k} \in \mathbb{R}^{2}} \sup _{\rho, E^{\mathrm{eq}}\left[\rho^{2} \mid y_{k}\right]=1}\left[E^{\mathrm{eq}}\left[\frac{\lambda^{2}}{\beta} v_{0, k} \rho^{2}\right]-\frac{\beta N^{4}}{2 k+1} D_{\gamma_{y, k}^{c}}(\rho)\right] \\
&=\frac{1}{(2 k+1) k^{4}} \sup _{|\lambda| \leq M} \sup _{y_{k} \in \mathbb{R}^{2}} N^{4} \sup _{\rho, E^{\mathrm{eq}}\left[\rho^{2} \mid y_{k}\right]=1}\left[E^{\mathrm{eq}\left[\frac{\lambda^{2}(2 k+1) k^{4}}{N^{4} \beta} v_{0, k} \rho^{2}\right]}\right. \\
&\left.-\beta k^{4} D_{v_{y, k}^{c}}(\rho)\right] .
\end{aligned}
$$

Moreover, $v_{i, k}$ is a bounded function (see Lemma 5.2) and $E^{\mathrm{eq}}\left[v_{0, k} \mid y_{k}\right]=0$. We can apply Lemma 4.2,

$$
\limsup _{N \rightarrow \infty} N^{4} \sup _{\rho, E^{\mathrm{eq}}\left[\rho^{2} \mid y_{k}\right]=1}\left[E^{\mathrm{eq}}\left[\frac{\lambda^{2}(2 k+1) k^{4}}{N^{4} \beta} v_{0, k} \rho^{2}\right]-\beta k^{4} D_{v_{y, k}^{c}}(\rho)\right] \leq 0 .
$$

The convergence on the line above is uniform over all possible values of the conserved quantities $y_{l}=\left(y_{l}^{1}, y_{l}^{2}\right)$; therefore $\lim \sup _{N \rightarrow \infty} \operatorname{supspec}_{L^{2}\left(\nu_{N}^{\text {eq }}\right)}\left(\Omega_{2}\right) \leq 0$.

The operator $\Omega_{3}$. We refer to Lemma 4.1. Rather than proving that

$$
\limsup _{\delta, r, l, k, N \rightarrow \infty} \operatorname{supspec}_{L^{2}\left(\nu_{N}^{\text {eq }}\right)}\left(\Omega_{3}\right)=0
$$


we will show that

$$
\limsup _{\delta, r, l, k, N \rightarrow \infty} \frac{1}{N} \log E^{\mathrm{eq}}\left[\exp \left(\int_{0}^{T} N \Omega_{3} d s\right)\right]=0
$$

where

$$
\Omega_{3}=\frac{1}{\beta N} \sum_{i=1}^{N} \phi\left(\frac{i}{N}\right)^{2} E^{\mathrm{eq}}\left[V_{l}\left(w-\hat{a}\left(\bar{x}_{l}\right) \delta x-L_{\infty} f_{r}, y\right) \mid y_{k}\right] \mathbf{1}_{\left|y_{k}\right| \geq \delta}
$$

Equation (4.57) follows from the estimations

$$
\begin{aligned}
\frac{1}{N} \log E^{\mathrm{eq}}\left[\exp \left(N \int_{0}^{T} \Omega_{3} d s\right)\right] \\
\quad \leq \frac{1}{N} \log E^{\mathrm{eq}}\left[\frac{1}{T} \int_{0}^{T} \exp \left(N T \Omega_{3}\right) d s\right] \\
\quad=\frac{1}{N} \log E^{\mathrm{eq}}\left[\exp \left(\sum_{i=1}^{N} \phi\left(\frac{i}{N}\right)^{2} E^{\mathrm{eq}}\left[\mathbb{V}_{l}\left(w-\hat{a}\left(\bar{x}_{l}\right) \Delta x-L_{\infty} f_{r}, y\right) \mid y_{k}\right] \mathbf{1}_{\left|y_{k}\right| \geq \delta}\right)\right] \\
\quad \leq \log E_{\gamma_{0}^{\mathrm{gc}}}\left[\exp \left(M^{2} E^{\mathrm{eq}}\left[\mathbb{V}_{l}\left(w-\hat{a}\left(\bar{x}_{l}\right) \Delta x-L_{\infty} f_{r}, y\right) \mid y_{k}\right] \mathbf{1}_{\left|y_{k}\right| \geq \delta}\right)\right] .
\end{aligned}
$$

The dominated convergence theorem can be applied (see the note at the end of Section 5) and gives us

$$
\lim _{\delta \rightarrow \infty} \log E_{\gamma_{0}^{\mathrm{gc}}}\left[\exp \left(M^{2} E^{\mathrm{eq}}\left[\mathbb{V}_{l}\left(w-\hat{a}\left(\bar{x}_{l}\right) \Delta x-L_{\infty} f_{r}, y\right) \mid y_{k}\right] \mathbf{1}_{\left|y_{k}\right| \geq \delta}\right)\right]
$$

The operator $\Omega_{4}$. The main purpose of Section 5 is to show that there exists a sequence of functions $\left\{f_{r}\right\}_{r \geq 0}$ such that for any $\delta>0$,

$$
\begin{gathered}
\limsup _{r, l, k \rightarrow \infty} \sup _{\left|y_{k}\right| \leq \delta} E^{\mathrm{eq}}\left[\mathbb{V}_{l}\left(w-\hat{a}\left(\bar{x}_{l}\right) \Delta x-L_{\infty} f_{r}, y\right) \mid y_{k}\right]=0, \\
\quad \sup _{r} \sup _{y_{k} \in \mathbb{R}^{2}} E^{\mathrm{eq}}\left[\mathbb{V}_{l}\left(w-\hat{a}\left(\bar{x}_{l}\right) \Delta x-L_{\infty} f_{r}, y\right) \mid y_{k}\right] \leq \infty
\end{gathered}
$$

The term (4.8). It follows that the term (4.8) converges to zero in probability from the following two lemmas. We refer the reader to Bertini et al. [2] or Guo et al. [8] for the proof. The proof uses mainly the entropy inequality (3.5) and the consequence of Feynman-Kac formula (4.18).

Lemma 4.6 (local ergodicity). Let $f$ be a cylinder function. Define $\tilde{f}: \mathbb{R} \rightarrow \mathbb{R}$ to be the function $\tilde{f}(\alpha)=E_{\gamma_{\alpha}^{\mathrm{gc}}}[f]$. Then for any $\delta>0, \phi: \mathbb{T} \rightarrow \mathbb{R}$ a smooth function,

$$
\underset{k, N \rightarrow \infty}{\limsup } P_{N, T}^{\mathrm{neq}}\left\{\int_{0}^{t}\left|\frac{1}{N} \sum_{i=1}^{N} \phi\left(\frac{i}{N}\right)\left(\tau^{i} f(x(s))-\tilde{f}\left(\mathrm{Av}_{j=i-k}^{i+k} x_{j}(s)\right)\right)\right| d s \geq \delta\right\}=0 .
$$


Lemma 4.7 (two-block estimate). For any continuous function $g: \mathbb{R} \rightarrow \mathbb{R}$, let

$$
F_{k, a, N}=\left\{\int_{0}^{t} \operatorname{Av}_{i=1}^{N} A v_{j=i-a N}^{i+a N}\left(g\left(\operatorname{Av}_{l=i-k}^{i+k} x_{l}(s)\right)-g\left(\operatorname{Av}_{l=j-k}^{j+k} x_{l}(s)\right)\right)^{2} d s \geq \delta\right\} .
$$

Then for any $\delta>0$,

$$
\limsup _{k \rightarrow \infty, a \rightarrow 0, N \rightarrow \infty} P_{N, T}^{\text {neq }}\left\{F_{k, a, N}\right\}=0 .
$$

\section{Computation of the central limit theorem variances}

In this section, we compute the value of the limit

$$
\limsup _{l \rightarrow \infty} \sup _{|\alpha| \leq \delta} E_{\gamma_{\alpha}^{\mathrm{gc}}}\left[\mathbb{V}_{l}(f, y)\right]
$$

for a particular class of cylinder functions $f$ to be described later.

We will need a result that relates canonical and grand canonical measures, known as the equivalence of ensemble. The equivalence of ensemble says that asymptotically the marginal in a fixed box of the canonical measure is the marginal of the grand canonical measure.

Lemma 5.1 (equivalence of ensemble). Let $f\left(x_{-s}, \ldots, x_{s}\right)$ be a bounded, local function. Then, for any $\epsilon>0$, there exist $N \in \mathbb{N}, \delta>0$, and $M>0$ such that

$$
\left|E_{\gamma_{y, k}^{c}}[f]-E_{\gamma_{\alpha}^{\mathrm{gc}}}[f]\right| \leq \epsilon
$$

as long as $k>N$ and $\left|y_{k}^{1}-\alpha\right|<\delta,\left|y_{k}^{2}\right|<M$. Assume that the potential $V(x)=x^{2} / 2$.

Proof. To keep the computations at minimum, we assume that the local function $f$ depends only on two coordinates $x_{i}$ and $x_{j}$. Since the potential $V(x)=x^{2} / 2$, the canonical measure $v_{y, k}^{c}$ is a multidimensional Gaussian probability measure. We will identify below the marginals of $v_{y, k}^{c}$ as Gaussian probability measures with certain means and variances.

Recall that

$$
y_{k}^{1}=\frac{1}{2 k+1} \sum_{i=-k}^{k} x_{i}, \quad y_{k}^{2}=\frac{1}{k(k+1)} \sum_{i=-k}^{k} i x_{i},
$$

and that $\nu_{y, k}^{c}$ is the conditional probability measure $\nu_{\beta, k}^{\mathrm{gc}}\left(\cdot \mid y_{k}^{1}, y_{k}^{2}\right)$. We can take $\beta=0$.

Under the grand canonical measure $v_{0, k}^{\mathrm{gc}}$, the random variables $x_{-k}, \ldots, x_{k}$ are independent and identically standard Gaussian distributed. Then, the covariance matrix of the random variables $\left(x_{i}, y_{k}^{1}, y_{k}^{2}\right)$ can be found to be

$$
\Sigma\left(x_{i}, y_{k}^{1}, y_{k}^{2}\right)=\left(\begin{array}{ccc}
1 & \frac{1}{2 k+1} & \frac{i}{k(k+1)} \\
\frac{1}{2 k+1} & \frac{1}{2 k+1} & 0 \\
\frac{i}{k(k+1)} & 0 & \frac{2 k+1}{3 k(k+1)}
\end{array}\right),
$$


and the conditional expectation

$$
E\left[x_{i} \mid y_{k}^{1}, y_{k}^{2}\right]=\frac{3 k^{2}(k+1)^{2}(2 k+1)}{2 k^{2}(k+1)-3 i^{2}}\left[\frac{1}{3 k(k+1)} y_{k}^{1}+\frac{i}{k(k+1)(2 k+1)} y_{k}^{2}\right]
$$

It is not difficult to observe that if $\left\{x_{i}\right\}_{i \in \mathbb{Z}}$ is a double-sided sequence such that $y_{k}^{1} \rightarrow \alpha$ as $k \rightarrow \infty$ and $\left\{y_{k}^{2}\right\}_{k \geq 1}$ is bounded, then

$$
E\left[x_{i} \mid y_{k}^{1}, y_{k}^{2}\right] \longrightarrow \alpha \text { as } k \longrightarrow \infty
$$

Moreover the conditional variance and covariance have the properties

$$
\begin{gathered}
E\left[\left(x_{i}-E\left(x_{i} \mid y_{k}^{1}, y_{k}^{2}\right)\right)^{2} \mid y_{k}^{1}, y_{k}^{2}\right]=\frac{2 k^{2}(k+1)-3 i^{2}}{k(k+1)(2 k+1)} \longrightarrow 1, \quad \text { as } k \rightarrow \infty, \\
E\left[\left(x_{i}-E\left(x_{i} \mid y_{k}^{1}, y_{k}^{2}\right)\right)\left(x_{j}-E\left(x_{j} \mid y_{k}^{1}, y_{k}^{2}\right)\right) \mid y_{k}^{1}, y_{k}^{2}\right] \\
=\frac{[k(k+1)+3 i j]\left[k(k+1)\left(4 k^{2}-1\right)+k\left(6 i^{2}+6 j^{2}\right)-6 i j\right]}{(2 k+1)\left[\left(2 k^{2}(k+1)-3 j^{2}\right)\left(2 k^{2}(k+1)-3 i^{2}\right)-k(k+1)-3 i j\right]} \longrightarrow 0 \text { as } k \longrightarrow \infty .
\end{gathered}
$$

The convergences exhibited above lead us to conclude that under the assumption that $y_{k}^{1} \rightarrow \alpha$ as $k \rightarrow \infty$ and that there exists $M$ such that $\left|y_{k}^{2}\right| \leq M, k \geq 1$, the marginal in a finite box of the canonical measure $v_{y, k}^{c}$ converges towards the marginal of the grand canonical measure $v_{\alpha}^{\mathrm{gc}}$ as $k \rightarrow \infty$. This convergence is in the sense explained in the statement of this lemma. The lemma follows. It is very possible that the techniques outlined in [8] or [11] can establish this lemma for a more general potential $V(x)$.

We will need a new notation, namely, $X_{0}^{*}$ for the adjoint of the vector field $X_{0}=\partial_{-1}-$ $2 \partial_{0}+\partial_{1}$ with respect to the inner product of the Hilbert space $L^{2}\left(a d \nu_{N}^{\mathrm{eq}}\right)$. The adjoint is given by the formula

$$
X_{0}^{*}(h)=-X_{0}(a h)+\left(V^{\prime}\left(x_{-1}\right)-2 V^{\prime}\left(x_{0}\right)+V^{\prime}\left(x_{1}\right)\right) a h .
$$

Note that the current $w$ is equal to $X_{0}^{*}(-1)$, and the slope Laplacian $\Delta x$ is equal to $X_{0}^{*}(1 / a)$.

LEMMA 5.2. Let $h\left(x_{-s}, \ldots, x_{s}\right)$ be a bounded, cylinder function such that $f=X_{0}^{*}(h) \in L^{2}\left(\nu_{\alpha, s}^{\mathrm{gc}}\right)$ for all $\alpha \in \mathbb{R}$. Then there exists $C(h)<\infty$ that depends just on the function $h$ such that

$$
\sup _{l, y_{0, l} \in \mathbb{R}^{2}} \frac{1}{l}\left\langle\left(-L_{l}\right)^{-1}\left(\sum_{|j| \leq l-\sqrt{l}} \tau^{j} f\right), \sum_{|i| \leq l-\sqrt{l}} \tau^{i} f\right\rangle \leq C(h) .
$$


In addition if the function $h$ is bounded away from zero, $h \geq C>0$, there exists the lower bound $C_{1}(h)>0$ that depends just on $h$,

$$
C_{1}(h) \leq \sup _{l, y_{0, l} \in \mathbb{R}^{2}} \frac{1}{l}\left\langle\left(-L_{l}\right)^{-1}\left(\sum_{|j| \leq l-\sqrt{l}} \tau^{j} f\right), \sum_{|i| \leq l-\sqrt{l}} \tau^{i} f\right\rangle .
$$

Proof. Remember that $\mathbb{V}_{l}(f, y)=(1 / l)\left\langle\left(-L_{l}\right)^{-1}\left(\sum_{|j| \leq l-\sqrt{l}} \tau^{j} f\right), \sum_{|i| \leq l-\sqrt{l}} \tau^{i} f\right\rangle_{\nu_{y, l}^{c}}$. We use the variational formula

$$
\mathbb{V}_{l}(f, y)=\sup _{u} \frac{\left\langle u, \sum_{|j| \leq l-\sqrt{l}} \tau^{j} f\right\rangle_{v_{y, l}^{c}}^{2}}{l D_{\gamma_{y, l}^{c}}(u)}
$$

We have

$$
\begin{aligned}
\left\langle u, \sum_{|j| \leq l-\sqrt{l}} \tau^{j} f\right\rangle_{v_{y, l}^{c}}^{2} & =\left\langle u, \sum_{|j| \leq l-\sqrt{l}} X_{j}^{*}\left(\tau^{j} h\right)\right\rangle_{\gamma_{y, l}^{c}}^{2}=E_{\gamma_{y, l}^{c}}\left[\sum_{|j| \leq l-\sqrt{l}} a_{j} X_{j}(u) \tau^{j} h\right]^{2} \\
& \leq D_{\gamma_{y, l}^{c}}(u) E_{\gamma_{y, l}^{c}}\left[\sum_{|j| \leq l-\sqrt{l}} a_{j}\left(\tau^{j} h\right)^{2}\right] \leq 2 l C(h) D_{\gamma_{y, l}^{c}}(u),
\end{aligned}
$$

therefore the upper bound for the variance is established.

For the lower bound, we may choose a particular function $u$ in (5.11), namely, $u=$ $\sum_{i=-l}^{l}\left(i^{2} / 2\right) x_{i}$. Note that $X_{i}(u)=1$ for any $-l+1 \leq i \leq l-1$. It follows that

$$
\frac{\left\langle u, \sum_{|j| \leq l-\sqrt{l}} \tau^{j} f\right\rangle_{\gamma_{y, l}^{c}}^{2}}{l D_{\gamma_{y, l}^{c}}(u)}=\frac{E_{\gamma_{y, l}^{c}}\left[\sum_{|j| \leq l-\sqrt{l}} a_{j} \tau^{j} h\right]^{2}}{l E_{\gamma_{y, l}^{c}}\left[\sum_{|j| \leq l-1} a_{j}\right]} \geq C_{1}(h) .
$$

We have just used that $a$ and $h$ are functions bounded away from zero, and $a$ is a bounded function.

Note. Lemma 5.2 is true for any local function $f=\sum_{i=-s}^{s} X_{j}^{*}\left(h_{j}\right)$, where the functions $h_{j}$ are bounded, local functions.

Definition 5.3. For a bounded, local function $f$ such that $E_{\gamma_{y, l}^{c}}[f]=0$, for all possible values of $y \in \mathbb{R}^{2}$, define the seminorm

$$
\langle\langle f\rangle\rangle_{\alpha}^{2}=\limsup _{l \rightarrow \infty, y_{k}^{1} \rightarrow \alpha} \frac{1}{l} E_{\gamma_{y, k}^{c}}\left[\left\langle\left(-L_{l}\right)^{-1}\left(\sum_{|i| \leq l-\sqrt{l}} \tau^{i} f\right), \sum_{|j| \leq l-\sqrt{l}} \tau^{j} f\right\rangle_{\gamma_{y, l}^{c}}\right] .
$$


We saw in Lemma 5.2 that $\langle\langle f\rangle\rangle_{\alpha}$ is a finite number as long as $f$ is equal to $X_{0}^{*}(h)$, where $h$ is a bounded, local function. By polarization, we can extend the seminorm $\langle\langle\cdot\rangle\rangle_{\alpha}$ to a semi-inner product $\langle\langle\cdot, \cdot\rangle\rangle_{\alpha}$. For the remaining part of this section, we compute the value of the seminorm $\langle\langle f\rangle\rangle_{\alpha}$ for certain function $f$.

Lemma 5.4. Assume that $g$ is a bounded cylinder function with bounded first derivatives, $f=L_{\infty} g, w=X_{0}(a)-\left(V^{\prime}\left(x_{-1}\right)-2 V^{\prime}\left(x_{0}\right)+V^{\prime}\left(x_{1}\right)\right) a$, and $\Delta x=x_{-1}-2 x_{0}+x_{1}$, then the following identities hold:

(a) $\left\langle\left\langle L_{\infty} g\right\rangle\right\rangle_{\alpha}^{2}=E_{\gamma_{\alpha}^{\mathrm{gc}}}\left[a\left(x_{-1}, x_{0}, x_{1}\right)\left(X_{0}\left(\sum_{j \in \mathbb{Z}} \tau^{j} g\right)\right)^{2}\right]$,

(b) $\langle\langle w\rangle\rangle_{\alpha}^{2}=4 E_{\gamma_{\alpha}^{\mathrm{gc}}}\left[a\left(x_{-1}, x_{0}, x_{1}\right)\right]$,

(c) $\left\langle\left\langle L_{\infty} g, w\right\rangle\right\rangle_{\alpha}=2 E_{\gamma_{\alpha}^{g c}}\left[a\left(x_{-1}, x_{0}, x_{1}\right) X_{0}\left(\sum_{j \in \mathbb{Z}} \tau^{j} g\right)\right]$,

(d) $\left\langle\left\langle L_{\infty} g, \Delta x\right\rangle\right\rangle_{\alpha}=0$,

(e) $\langle\langle w, \Delta x\rangle\rangle_{\alpha}=4$.

Proof. One checks directly using equivalence of ensemble Lemma 5.1 and the asymptotic shift invariance of $v_{y, k}^{c}$ that the relations (a)-(e) hold. In particular, for (d) and (e), it is important to notice that $\Delta x=x_{-1}-2 x_{0}+x_{1}=X_{0}^{*}(1 / a)$.

Definition 5.5. Define the Hilbert space $\mathscr{H}_{\alpha}$ to be the closed linear span in $L^{2}\left(a d \nu_{\alpha}^{\text {gc }}\right)$ of the function 1 and functions $\xi_{g}=X_{0}\left(\sum_{j \in \mathbb{Z}} \tau^{j} g\right)$, where $g$ is a bounded local function with bounded first derivatives.

It is not hard to see that if $f$ is equal to $X_{0}^{*}(h)$, then

$$
\begin{gathered}
\left\langle\left\langle f, L_{\infty} g\right\rangle\right\rangle_{\alpha}=E_{\gamma_{\alpha}^{\mathrm{gc}}}\left[a \operatorname{Proj}_{\mathscr{H}_{\alpha}}(2 h) X_{0}\left(\sum_{j \in \mathbb{Z}} \tau^{j} g\right)\right], \\
\langle\langle f, w\rangle\rangle_{\alpha}=E_{\gamma_{\alpha}^{\mathrm{gc}}}\left[a \operatorname{Proj}_{\mathcal{H}_{\alpha}}(2 h) 2\right] .
\end{gathered}
$$

On both lines above, Proj $\dot{\mathscr{H}}_{\alpha}$ stands for the projection operator in the subspace $\mathscr{H}_{\alpha}$. We are left to calculate $\langle\langle f\rangle\rangle_{\alpha}$ for a function $f=X_{0}^{*}(h)$. As we will show in the following lemma, $\langle\langle f\rangle\rangle_{\alpha}^{2}=E_{\gamma_{\alpha}^{g} c}\left[a\left(\operatorname{Proj}_{\mathscr{H}_{\alpha}}(2 h)\right)^{2}\right]$.

Lemma 5.6. Suppose that $f$ is equal to $X_{0}^{*}(h)$, where $h$ is a bounded cylinder function. Then the following holds:

$$
\langle\langle f\rangle\rangle_{\alpha}^{2}=E_{\gamma_{\alpha}^{g c}}\left[a\left(\operatorname{Proj}_{\mathscr{H}_{\alpha}}(2 h)\right)^{2}\right]
$$

Proof. Using Cauchy-Schwarz inequality, it follows that

$$
\begin{aligned}
\langle\langle f\rangle\rangle_{\alpha}^{2} & =\liminf _{l \rightarrow \infty, y_{k}^{1} \rightarrow \alpha} \frac{1}{l} E_{\gamma_{y, k}^{c}}\left[\left\langle\left(-L_{l}\right)^{-1}\left(\sum_{|i| \leq l-\sqrt{l}} \tau^{i} f\right), \sum_{|j| \leq l-\sqrt{l}} \tau^{j} f\right\rangle\right] \\
& \geq E_{\gamma_{\alpha}^{\mathrm{gc}}}\left[a\left(\operatorname{Proj}_{\gamma_{\alpha, l}^{c}}(2 h)\right)^{2}\right] .
\end{aligned}
$$


Consider $g=\sum_{|j| \leq l-\sqrt{l}} \tau^{j} f$, where $f=X_{0}^{*}(h)$, then

$$
\begin{aligned}
\frac{1}{l}\langle( & \left.\left.-L_{l}\right)^{-1}\left(\sum_{|i| \leq l-\sqrt{l}} \tau^{i} f\right), \sum_{|j| \leq l-\sqrt{l}} \tau^{j} f\right\rangle \\
= & \sup _{\rho, D_{\gamma_{y, l}^{c}}(\rho)=2 l} \frac{\left\langle\rho, \sum_{|j| \leq l-\sqrt{l}} \tau^{j} f\right\rangle_{\gamma_{y, l}^{c}}^{2}}{2 l^{2}}=\frac{\left\langle\rho_{l}, \sum_{|j| \leq l-\sqrt{l}} \tau^{j} f\right\rangle_{\gamma_{y, l}^{c}}^{2}}{2 l^{2}} \\
= & \frac{\left\langle\rho_{l}, \sum_{|j| \leq l-\sqrt{l}} X_{j}^{*}\left(\tau^{j} h\right)\right\rangle_{\gamma_{y, l}^{c}}^{2}}{2 l^{2}}=\frac{1}{2 l^{2}} E_{\gamma_{y, l}^{c}}\left[\sum_{|j| \leq l-\sqrt{l}} a\left(x_{-j}, x_{j}, x_{j}\right) X_{j}\left(\rho_{l}\right) \tau^{j} h\right]^{2} .
\end{aligned}
$$

Above, $\rho_{l}$ is some maximizer function with $D_{\gamma_{y, l}^{c}}\left(\rho_{l}\right)=2 l$.

Define $u_{l}=(1 / 2 l) \sum_{|j| \leq l-\sqrt{l}} \tau^{-j}\left(X_{j} \rho_{l}\right)$. We have

$$
E_{\gamma_{\alpha}^{\mathrm{gc}}}\left[a\left(u_{l}\right)^{2}\right] \leq E_{\gamma_{\alpha}^{\mathrm{gc}}}\left[\frac{a}{2 l} \sum_{|j| \leq l-\sqrt{l}}\left(\tau^{-j}\left(X_{j} \rho_{l}\right)\right)^{2}\right]=\frac{1}{l} D_{\gamma_{\alpha}^{\mathrm{gc}}}\left(\rho_{l}\right) \leq 2 .
$$

The above inequality shows that $\left\{u_{l}\right\}_{l}$ is a bounded sequence in $L^{2}\left(a d \nu_{\alpha}^{\mathrm{gc}}\right)$, and hence has a weakly convergent subsequence in $L^{2}\left(a d v_{\alpha}^{\mathrm{gc}}\right)$. Let $u$ be the weak limit of a convergent subsequence of $\left\{u_{l}\right\}_{l}$. It follows that

$$
\begin{aligned}
\lim \sup _{l \rightarrow \infty, y_{k}^{1} \rightarrow \alpha} & \left.\frac{1}{l} E_{\gamma_{y, k}^{c}}\left[\left\langle\left(-L_{l}\right)^{-1}\left(\sum_{|i| \leq l-\sqrt{l}} \tau^{i} f\right), \sum_{|j| \leq l-\sqrt{l}} \tau^{j} f\right\rangle\right\rangle_{\gamma_{y, l}^{c}}\right] \\
\leq & 2 E_{\gamma_{\alpha}^{\mathrm{gc}}}[a u h]^{2}=2 E_{\gamma_{\alpha}^{\mathrm{gc}}}\left[a u \operatorname{Proj}_{\mathcal{H}_{\alpha}} h\right]^{2} \\
\leq & 2 E_{\gamma_{\alpha}^{\mathrm{gc}}}\left[a u^{2}\right] E_{\gamma_{\alpha}^{\mathrm{gc}}}\left[a\left(\operatorname{Proj}_{\mathcal{H}_{\alpha}} h\right)^{2}\right] \leq E_{\gamma_{\alpha}^{\mathrm{gc}}}\left[a\left(\operatorname{Proj}_{\mathscr{H}_{\alpha}} 2 h\right)^{2}\right] .
\end{aligned}
$$

The key point that has allowed us to write the above inequalities is that the function $u$ has the property $X_{a}\left(\tau^{b} u\right)=X_{b}\left(\tau^{a} u\right)$ for all integers $a$ and $b$, and a function with this property belongs to $\mathscr{H}_{\alpha}$ (see Lemma 2.3 or Savu $\left.[16,17]\right)$. The next estimate shows that $X_{a}\left(\tau^{b} u\right)=X_{b}\left(\tau^{a} u\right)$ is valid in a weak sense. Consider a smooth test function $\phi$, with bounded first derivatives. Assume $a>b$. We have

$$
\begin{gathered}
\left\langle X_{a}\left(\tau^{b} u_{l}\right), \phi\right\rangle_{\nu_{\alpha}}=\left\langle\frac{1}{2 l} \sum_{|j| \leq l-\sqrt{l}} \tau^{b-j}\left(\rho_{l}\right), X_{b}^{*} X_{a}^{*} \phi\right\rangle_{\gamma_{\alpha}^{\mathrm{gc}}}, \\
\left\langle X_{a}\left(\tau^{b} u_{l}\right)-X_{b}\left(\tau^{a} u_{l}\right), \phi\right\rangle_{\gamma_{\alpha}^{\mathrm{gc}}} \\
=\left\langle\frac{1}{2 l} \sum_{j=b+1+l-\sqrt{l}}^{a+l-\sqrt{l}} \tau^{j}\left(\rho_{l}\right)-\frac{1}{2 l} \sum_{j=b-l+\sqrt{l}}^{a-1-l+\sqrt{l}} \tau^{j}\left(\rho_{l}\right), X_{b}^{*} X_{a}^{*} \phi\right\rangle_{\gamma_{\alpha}^{\mathrm{gc}}} \\
=\left\langle\frac{1}{2 l} \sum_{j=b+1+l-\sqrt{l}}^{a+l-\sqrt{l}} \tau^{j} X_{b-j}\left(\rho_{l}\right)+\frac{1}{2 l} \sum_{j=b-l+\sqrt{l}}^{a-1-l+\sqrt{l}} \tau^{j} X_{b-j}\left(\rho_{l}\right), X_{a}^{*} \phi\right\rangle_{\gamma_{\alpha}^{\mathrm{gc}}} .
\end{gathered}
$$


By Cauchy-Schwarz inequality, we obtain

$$
\begin{aligned}
& \left\langle X_{a}\left(\tau^{b} u_{l}\right)-X_{b}\left(\tau^{a} u_{l}\right), \phi\right\rangle_{\gamma_{\alpha}^{\mathrm{gc}}}^{2} \\
& \leq E_{\gamma_{\alpha}^{\mathrm{gc}}}\left[\left(\frac{1}{2 l} \sum_{j=b+1+l-\sqrt{l}}^{a+l-\sqrt{l}} \tau^{j} X_{b-j}\left(\rho_{l}\right)+\frac{1}{2 l} \sum_{j=b-l+\sqrt{l}}^{a-1-l+\sqrt{l}} \tau^{j} X_{b-j}\left(\rho_{l}\right)\right)^{2}\right] E_{\gamma_{\alpha}^{\mathrm{gc}}}\left[\left(X_{a}^{*} \phi\right)^{2}\right] \\
& \leq \frac{C(a-b)}{(2 l)^{2}}\left(\sum_{j=b-a-l+\sqrt{l}}^{-1-l+\sqrt{l}} E_{\gamma_{\alpha}^{\mathrm{gc}}}\left[\left(X_{j} \rho_{l}\right)^{2}\right]+\sum_{j=b-a+1+l-\sqrt{l}}^{l-\sqrt{l}} E_{\gamma_{\alpha}^{\mathrm{gc}}}\left[\left(X_{j} \rho_{l}\right)^{2}\right]\right) \\
& \leq \frac{C(a-b)}{(2 l)^{2}} D_{\gamma_{\alpha}^{\mathrm{gc}}}\left(\rho_{l}\right) \leq \frac{C(a-b)}{2 l} .
\end{aligned}
$$

As $l$ converges to infinity, the sequence $\left\{u_{l}\right\}_{l}$ approaches $u$ in the weak sense. Combining this fact with (5.22), we can establish $X_{a}\left(\tau^{b} u\right)=X_{b}\left(\tau^{a} u\right)$.

Now we can conclude that if we have a local function $f$ such that $f=X_{0}^{*}(h)$, then

$$
\begin{aligned}
\langle\langle f, f\rangle\rangle_{\alpha} & \left.=\lim _{l \rightarrow \infty, y_{k}^{1} \rightarrow \alpha} \frac{1}{l} E_{\gamma_{y, k}^{c}}\left[\left\langle\left(-L_{l}\right)^{-1}\left(\sum_{|i| \leq l-\sqrt{l}} \tau^{i} f\right), \sum_{|j| \leq l-\sqrt{l}} \tau^{j} f\right\rangle\right\rangle_{\gamma_{y, l}^{c}}\right] \\
& =E_{\gamma_{\alpha}^{\mathrm{gc}}}\left[a\left(\operatorname{Proj}_{\mathscr{H}_{\alpha}}(2 h)\right)^{2}\right] .
\end{aligned}
$$

The results proved so far in this section allow us to conclude that if $g$ is a local function equal to $X_{0}^{*}(h)$ and $b\left(y_{k}^{1}\right)$ is a coefficient that depends on the mean slope in a box of size $k$, then

$$
\begin{aligned}
& \left\langle\left\langle g+b w+L_{\infty} f, g+b w+L_{\infty} f\right\rangle\right\rangle_{\alpha} \\
& =\lim _{l \rightarrow \infty, y_{k}^{1} \rightarrow \alpha} E_{\gamma_{y, k}^{c}}\left[\mathbb{V}_{l}\left(g+b w+L_{\infty} f, y\right)\right] \\
& =E_{a \gamma_{\alpha}^{\mathrm{gc}}}\left[\left(\operatorname{Proj}_{\mathcal{H}_{\alpha}}(2 h)+2 b+X_{0}\left(\sum_{i \in \mathbb{Z}} \tau^{i} f\right)\right)^{2}\right] .
\end{aligned}
$$

Since Proj $\dot{\mathscr{H}}_{\alpha}(2 h)$ is a function in the closed linear span of the functions 1 and $X_{0}\left(\sum_{i \in \mathbb{Z}} \tau^{i} f\right)$, where $f$ is a local function, there follows the existence of a sequence of functions $\left\{f_{r}\right\}_{r>0}$ and of a coefficient $b=1 / \hat{a}$ such that

$$
\lim _{r \rightarrow \infty}\left\langle\left\langle\Delta x+b w+L_{\infty} f_{r}\right\rangle\right\rangle_{\alpha}=0 .
$$


Lemma 5.7. Let $a^{*}>0$ be an upper bound for the function $a$. For any $\epsilon>0$ and $\delta>0$, there exists a smooth function $f\left(x_{-l}, \ldots, x_{l}, \alpha\right)$ such that

$$
\begin{gathered}
\sup _{|\alpha| \leq \delta}\left\langle\left\langle w-L_{\infty} f-\hat{a}(\alpha) \Delta x\right\rangle\right\rangle_{\alpha}<\epsilon, \\
\sup _{\alpha}\left\langle\left\langle w-L_{\infty} f-\hat{a}(\alpha) \Delta x\right\rangle\right\rangle_{\alpha}<2 a^{*} .
\end{gathered}
$$

Proof. We calculate

$$
\left\langle\left\langle w-L_{\infty} f-\hat{a}(\alpha) \Delta x\right\rangle\right\rangle_{\alpha}^{2}=4\left[E_{\gamma_{\alpha}^{\mathrm{gc}}}\left[a\left(1+X_{0}\left(\sum_{i \in \mathbb{Z}} \tau^{i} f\right)\right)^{2}\right]-\hat{a}(\alpha)\right] .
$$

Let us introduce the notation $\mathscr{F}_{l, B}$ for the set of cylinder functions $f\left(x_{-l}, \ldots, x_{l}\right)$ with $\|f\|_{\infty} \leq B$ and $\left\|\partial_{i} f\right\|_{\infty} \leq B$ for $i=-l, \ldots, l$. Let $A(f, \alpha)=E_{\gamma_{\alpha}^{\mathrm{gc}}}\left[a\left(1+X_{0}\left(\sum_{i \in \mathbb{Z}} \tau^{i} f\right)\right)^{2}\right]-$ $\hat{a}(\alpha)$ and let $A_{l, B}(\alpha)=\inf _{f \in \mathscr{F}_{l, B}} A(f, \alpha)$. The function $A_{l, B}(\alpha)$ is upper semicontinuous and nonincreasing in $l$ and $B$, and for each $\alpha \in \mathbb{R}, \lim _{l, B \rightarrow \infty} A_{l, B}(\alpha)=0$, therefore

$$
\lim _{l, B \rightarrow \infty} \sup _{|\alpha| \leq \delta+1} A_{l, B}(\alpha)=0
$$

Therefore we can find for each $\alpha$ a cylinder function $f(\alpha)$ such that $A(f(\alpha), \alpha) \leq \epsilon$ if $|\alpha| \leq \delta+1$. We extend $f$ to be zero on $|\alpha|>\delta+1$. Then on $|\alpha|>\delta+1$, we have that $A(f(\alpha), \alpha)=E_{\gamma_{\alpha}^{\mathrm{gc}}}\left[a\left(x_{-1}, x_{0}, x_{1}\right)\right]-\hat{a}(\alpha)$ and $A(f(\alpha), \alpha) \leq a^{*}$. To be able to avoid the problem that occurs when $f$ is not smooth, we take the convolution of $f$ with a smoothing kernel $\phi$. The required function is the convolution $f * \phi$. For a complete argument, see Quastel [14, Lemma 2.6].

Note. From the equivalence of ensemble Lemma 5.1, we know that for any bounded cylinder function $f$ and any $\epsilon>0$, there exist $N_{1} \in \mathbb{N}$ and $\delta_{1}>0$ such that

$$
\left|E^{\mathrm{eq}}\left[\mathbb{V}_{l}\left(w-\hat{a}\left(\bar{x}_{i, l}\right) \Delta x-L_{\infty} f, y\right) \mid y_{k}\right]-\left\langle\left\langle w-\hat{a}(\alpha) \Delta x-L_{\infty} f\right\rangle\right\rangle_{\alpha}^{2}\right| \leq \epsilon,
$$

as long as $k>N_{1}, l>N_{1}$, and $\left|y_{k}^{1}-\alpha\right|<\delta_{1}$. Then Lemma 5.7 helps us to conclude that for any $\epsilon>0$, there exists a bounded cylinder function $f_{r}$ such that

$$
\begin{gathered}
\limsup \sup _{l, k \rightarrow \infty} E_{\left|y_{k}\right| \leq \delta}^{\mathrm{eq}}\left[\mathbb{V}_{l}\left(w-\hat{a}\left(\bar{x}_{l}\right) \Delta x-L_{\infty} f_{r}, y\right) \mid y_{k}\right]<2 \epsilon, \\
\sup _{y_{k} \in \mathbb{R}^{2}} E^{\mathrm{eq}}\left[\mathbb{V}_{l}\left(w-\hat{a}\left(\bar{x}_{l}\right) \Delta x-L_{\infty} f_{r}, y\right) \mid y_{k}\right] \leq 3 a^{*} .
\end{gathered}
$$

$a^{*}$ on the line above is the upper bound for the function $a$ in the hypothesis of Lemma 5.7. 
Properties of the transport coefficient. The transport coefficient $\hat{a}$ has been defined as the unique real number such that

$$
\inf _{f}\left\langle\left\langle w-\hat{a}(\alpha) \Delta x-L_{\infty} f\right\rangle\right\rangle_{\alpha}^{2}=0
$$

It is important to notice that the transport coefficient is also given by the formula

$$
\begin{aligned}
\hat{a}(\alpha) & =\frac{\langle\langle w, \Delta x\rangle\rangle_{\alpha}}{\langle\langle\Delta x, \Delta x\rangle\rangle_{\alpha}}=\frac{E_{\gamma_{\alpha}^{\mathrm{gc}}}[2(2 / a) a]}{E_{\gamma_{\alpha}^{\mathrm{gc}}}\left[\left(\operatorname{Proj}_{\mathcal{H}_{\alpha}}(2 / a)\right)^{2} a\right]}=\frac{1}{E_{\gamma_{\alpha}^{\mathrm{gc}}}\left[\left(\operatorname{Proj}_{\mathcal{H}_{\alpha}}(1 / a)\right)^{2} a\right]} \\
& =\frac{1}{\sup _{\gamma, g} E_{\gamma_{\alpha}^{\mathrm{gc}}}\left[\left(\operatorname{Proj}_{\gamma+X_{0}\left(\sum_{j \in \mathbb{Z}} \tau^{j} g\right)}(1 / a)\right)^{2} a\right]}=\inf _{\gamma, g} \frac{E_{\gamma_{\alpha}^{\mathrm{gc}}}\left[\left(\gamma+X_{0}\left(\sum_{j \in \mathbb{Z}} \tau^{j} g\right)\right)^{2} a\right]}{E_{\gamma_{\alpha}^{\mathrm{gc}}}\left[(1 / a)\left(\gamma+X_{0}\left(\sum_{j \in \mathbb{Z}} \tau^{j} g\right)\right) a\right]^{2}} \\
& =\inf _{g} E_{\gamma_{\alpha}^{\mathrm{gc}}}\left[a\left(x_{-1}, x_{0}, x_{1}\right)\left(1+X_{0}\left(\sum_{j \in \mathbb{Z}} \tau^{j} g\right)\right)^{2}\right] .
\end{aligned}
$$

\section{Hydrodynamic scaling limit of the model for surface electromigration}

In this section, we will show that the model for surface electromigration (2.12) has a hydrodynamic scaling limit as well. We will prove Theorem 2.4. The proof is inspired by Quastel [14]. From Cameron-Martin-Girsanov formula, we can find the Radon-Nikodym derivative

$$
\begin{aligned}
& \frac{d P_{N, E, T}^{\mathrm{neq}}}{d P_{N, T}^{\mathrm{eq}}}=\frac{d \nu_{N}^{\mathrm{neq}}}{d \nu_{N}^{\mathrm{eq}}} \exp \left(\frac{1}{2} \sum_{i=1}^{N} \int_{0}^{T} E\left(t, \frac{i}{N}\right) \sqrt{a\left(x_{i-1}, x_{i}, x_{i+1}\right)} d B_{i}\right. \\
& \left.-\frac{1}{8} \sum_{i=1}^{N} \int_{0}^{T} E^{2}\left(t, \frac{i}{N}\right) a\left(x_{i-1}, x_{i}, x_{i+1}\right) d t\right),
\end{aligned}
$$

and the relative entropy

$$
H\left(P_{N, E, T}^{\mathrm{neq}} \mid P_{N, T}^{\mathrm{eq}}\right)=H\left(v_{N}^{\mathrm{neq}} \mid v_{N}^{\mathrm{eq}}\right)+E_{P_{N, E, T}^{\mathrm{neq}}}\left[\frac{1}{8} \sum_{i=1}^{N} \int_{0}^{T} E^{2}\left(t, \frac{i}{N}\right) a\left(x_{i-1}, x_{i}, x_{i+1}\right) d t\right]
$$

Hence, there exists a constant $C$ such that $H\left(P_{N, E, T}^{\text {neq }} \mid P_{N, T}^{\mathrm{eq}}\right) \leq C N$. We can use the inequality (3.6) and the superexponential estimates (3.11) and (3.19) to conclude that the sequence of probability measures $\left\{Q_{N, E, T}^{\text {neq }}\right\}_{N \geq 0}$ is tight in the topology of the space $\mathscr{X}$. Denote by $Q_{E, T}$ a weak limit of a subsequence of $\left\{Q_{N, E, T}^{\text {neq }}\right\}_{N \geq 0}$. 
We proceed further to identify the limit $Q_{E, T}$. To save space, we ignore the time dependence of the test function $\phi$,

$$
\begin{aligned}
\frac{1}{N} \sum_{i=1}^{N} \phi & \left(\frac{i}{N}\right) x_{i}(T)-\frac{1}{N} \sum_{i=1}^{N} \phi\left(\frac{i}{N}\right) x_{i}(0) \\
= & \int_{0}^{T} \frac{1}{2 N} \sum_{i=1}^{N}\left(N^{2} w_{i}+E\left(t, \frac{i}{N}\right) a_{i}\right) \phi^{\prime \prime}\left(\frac{i}{N}\right) d t+M_{N}(T) \\
= & \int_{0}^{T} \frac{N^{2}}{2 N} \sum_{i=1}^{N}\left(w_{i}-\hat{a}\left(\bar{x}_{i, k}\right)(\Delta x)_{i}-L_{\infty} \tau^{i} f_{r}\right) \phi^{\prime \prime}\left(\frac{i}{N}\right) d t+M_{N}(T) \\
& +\int_{0}^{T} \frac{N^{2}}{2 N} \sum_{i=1}^{N} L_{N, E} \tau^{i} f_{r} \phi^{\prime \prime}\left(\frac{i}{N}\right) d t \\
& +\int_{0}^{T} \frac{1}{2 N} \sum_{i=1}^{N}\left[E\left(t, \frac{i}{N}\right)\left(a_{i}-\hat{a}\left(\bar{x}_{i, k}\right)\right)+N^{2}\left(L_{\infty} \tau^{i} f_{r}-L_{N, E} \tau^{i} f_{r}\right)\right] \phi^{\prime \prime}\left(\frac{i}{N}\right) d t \\
& +\int_{0}^{T} \frac{1}{2 N} \sum_{i=1}^{N} \hat{a}\left(\bar{x}_{i, k}\right)\left[N^{2}(\Delta x)_{i}+E\left(t, \frac{i}{N}\right)\right] \phi^{\prime \prime}\left(\frac{i}{N}\right) d t .
\end{aligned}
$$

Above $\left\{M_{N}(t)\right\}_{t \geq 0}$ is a martingale, and as in Section 4 we can prove that it is negligible in the limit. Because of the entropy inequality (3.5) and the superexponential estimate

$$
\begin{array}{r}
\limsup _{r, l, N \rightarrow \infty} \frac{1}{N} \log E^{\mathrm{eq}}\left[\operatorname { e x p } \left(\mid \int_{0}^{T} N^{2} \sum_{i=1}^{N}\left[\mathrm{Av}_{j=i-l_{1}}^{i+l_{1}} w_{j}-\hat{a}\left(\bar{x}_{i, l}\right) \operatorname{Av}_{j=i-l_{1}}^{i+l_{1}}(\Delta x)_{j}\right.\right.\right. \\
\left.\left.\left.-\mathrm{Av}_{j=i-l_{1}}^{i+l_{1}} \tau^{j} L_{\infty} f_{r}\right] d s \mid\right)\right] \leq 0
\end{array}
$$

the event on line (4.27) is negligible under $P_{N, E, T}^{\text {neq }}$. For the new model, we have that the negligible fluctuations are $L_{N, E} f$ and not $L_{\infty} f$ (i.e., the term (6.6) has no contribution towards the limit of the model). The contribution coming from nontrivial fluctuations $L_{N, E} f-L_{\infty} f$ are gathered in the coefficient in front of the vector field $E$ in the nonlinear equation (2.20). Because of this reason, the coefficient turns out to be the transport coefficient $\hat{a}$, defined by the variational formula (2.16) and not $\tilde{a}(\alpha)=E_{\gamma_{\alpha}^{\mathrm{gc}}}[a]$.

We will show that the sequence of smooth local functions $\left\{f_{r}\right\}_{r \geq 0}$ introduced in the note right after Lemma 5.7 can be chosen to have the additional property that the term (6.7) is negligible in the limit. Note that the term (6.7) is negligible if

$$
\limsup _{r, k, N \rightarrow \infty} P_{N, E, T}^{\text {neq }}\{|F(T)|>\epsilon\}=0
$$


where

$$
F(T)=\int_{0}^{T} \frac{1}{2 N} \sum_{i=1}^{N} E\left(t, \frac{i}{N}\right)\left[a_{i} g_{i}-\hat{a}\left(\bar{x}_{i, k}\right)\right] d t, \quad g_{i}=1+X_{i}\left(\sum_{j \in \mathbb{Z}} \tau^{j} f_{r}\right) .
$$

We can write

$$
\begin{aligned}
F(T)= & \int_{0}^{T} \frac{1}{2 N} \sum_{i=1}^{N} E\left(t, \frac{i}{N}\right)\left(a_{i} g_{i}-E_{\bar{y}_{i, k}^{\mathrm{gc}}}\left[a_{i} g_{i}\right]\right) \\
& +\int_{0}^{T} \frac{1}{2 N} \sum_{i=1}^{N} E\left(t, \frac{i}{N}\right)\left(E_{\gamma_{\bar{y}_{i}}^{\mathrm{gc}}}\left[a_{i} g_{i}\right]-\hat{a}\left(\bar{x}_{i, k}\right)\right) \mathbf{1}_{\left|\bar{y}_{i, k}^{1}\right| \leq \delta} \\
& +\int_{0}^{T} \frac{1}{2 N} \sum_{i=1}^{N} E\left(t, \frac{i}{N}\right)\left(E_{\gamma_{\bar{y}_{i, k}^{1}}^{\mathrm{gc}}}\left[a_{i} g_{i}\right]-\hat{a}\left(\bar{x}_{i, k}\right)\right) \mathbf{1}_{\left|\bar{y}_{i, k}^{1}\right| \geq \delta} .
\end{aligned}
$$

Recall that in the proof of Lemma 5.7, we introduced $A(f, \alpha)$ to be the difference $E_{\gamma_{\alpha}^{\mathrm{gc}}}[a(1+$ $\left.\left.X_{0}\left(\sum_{i \in \mathbb{Z}} \tau^{i} f\right)\right)^{2}\right]-\hat{a}(\alpha)$. We will show that we can modify the sequence $\left\{f_{r}\right\}_{r \geq 0}$ to have the properties

$$
\begin{gathered}
\sup _{\alpha, r} A\left(f_{r}(\alpha), \alpha\right) \leq a^{*}, \quad \sup _{\alpha, r}\left|E_{\gamma_{\alpha}^{\mathrm{gc}}}\left[a g_{0}\right]-\hat{a}(\alpha)\right| \leq 5 a^{*}, \\
\lim _{r \rightarrow \infty} \sup _{|\alpha| \leq \delta} A\left(f_{r}(\alpha), \alpha\right)=0, \\
\lim _{r \rightarrow \infty} \sup _{|\alpha| \leq \delta}\left|E_{\gamma_{\alpha}^{\mathrm{gc}}}\left[a g_{0}\right]-\hat{a}(\alpha)\right|=0 .
\end{gathered}
$$

We write

$$
\begin{aligned}
E_{\gamma_{\alpha}^{\mathrm{gc}}}\left[a g_{0}\right]-\hat{a}(\alpha) & =\left(E_{\gamma_{\alpha}^{\mathrm{gc}}}\left[a g_{0}\right]-E_{\gamma_{\alpha}^{\mathrm{gc}}}\left[a g_{0}^{2}\right]\right)+\left(E_{\gamma_{\alpha}^{\mathrm{gc}}}\left[a g_{0}^{2}\right]-\hat{a}(\alpha)\right) \\
& =E_{\gamma_{\alpha}^{\mathrm{gc}}}\left[a g_{0}\left(g_{0}-1\right)\right]+A\left(f_{r}(\alpha), \alpha\right) .
\end{aligned}
$$

Since $\left|E_{\gamma_{\alpha}^{\mathrm{gc}}}\left[a g_{0}\left(g_{0}-1\right)\right]\right| \leq 4 a^{*}$ and $A\left(f_{r}(\alpha), \alpha\right) \leq a^{*}$ uniform in $\alpha$ and $r,(6.13)$ follows. Consider $f^{*}(\alpha)$ to be the minimizer of $A(f, \alpha)$ among local functions of $x_{-l}$ through $x_{l}$. $f^{*}$ has the property that for any $f_{r}$,

$$
E_{\gamma_{\alpha}^{\mathrm{cc}}}\left[a\left(1+X_{0}\left(\sum_{j \in \mathbb{Z}} \tau^{j} f^{*}\right)\right) X_{0}\left(\sum_{j \in \mathbb{Z}} \tau^{j} f_{r}\right)\right]=0 .
$$

Now, thanks to Cauchy-Schwarz inequality,

$$
\begin{aligned}
\left|E_{\gamma_{\alpha}^{\mathrm{gc}}}\left[a g_{0}\left(g_{0}-1\right)\right]\right| & =\left|E_{\gamma_{\alpha}^{\mathrm{gc}}}\left[a X_{0}\left(\sum_{j \in \mathbb{Z}} \tau^{j} f_{r}\right) X_{0}\left(\sum_{j \in \mathbb{Z}} \tau^{j}\left(f^{*}-f_{r}\right)\right)\right]\right| \\
& \leq E_{\gamma_{\alpha}^{\mathrm{gc}}}\left[a\left(X_{0}\left(\sum_{j \in \mathbb{Z}} \tau^{j} f_{r}\right)\right)^{2}\right]^{1 / 2} E_{\gamma_{\alpha}^{\mathrm{gc}}}\left[a\left(X_{0}\left(\sum_{j \in \mathbb{Z}} \tau^{j}\left(f^{*}-f_{r}\right)\right)\right)^{2}\right]^{1 / 2} .
\end{aligned}
$$


We modify $f_{r}$ such that

$$
\sup _{|\alpha| \leq \delta} E_{\gamma_{\alpha}^{\mathrm{gc}}}\left[a\left(X_{0}\left(\sum_{j \in \mathbb{Z}} \tau^{j}\left(f^{*}-f_{r}\right)\right)\right)^{2}\right] \leq \frac{\epsilon^{2}}{6 a^{*}} .
$$

To conclude, (6.13) is negligible because of local ergodicity (Lemma 4.6), (6.14) is negligible because for any fixed $\delta$ we have (6.15), and (6.15) shrinks to zero once $\delta$ becomes very large. Also it is important to have property (6.13). Thus our result follows.

\section{Acknowledgments}

I thank Jeremy Quastel for proposing me the problem discussed in this paper. I thank George A. Elliott for many ideas and inspiring discussions that helped me to prove Lemma 2.3. This research is supported by an Ontario Graduate Studies Fellowship and by the University of Toronto Fellowship.

\section{References}

[1] A. Barabási and H. E. Stanley, Fractal Concepts in Surface Growth, Cambridge University Press, Cambridge, 1995.

[2] L. Bertini, C. Landim, and S. Olla, Derivation of Cahn-Hilliard equations from Ginzburg-Landau models, Journal of Statistical Physics 88 (1997), no. 1-2, 365-381.

[3] C. C. Chang and H.-T. Yau, Fluctuations of one-dimensional Ginzburg-Landau models in nonequilibrium, Communications in Mathematical Physics 145 (1992), no. 2, 209-234.

[4] J.-D. Deuschel and D. W. Stroock, Large Deviations, Pure and Applied Mathematics, vol. 137, Academic Press, Massachusetts, 1989.

[5] S. D. Eidel'man, Parabolic Systems, North-Holland, Amsterdam, 1969.

[6] J. Fritz, On the hydrodynamic limit of a scalar Ginzburg-Landau lattice model: the resolvent approach, Hydrodynamic Behavior and Interacting Particle Systems (Minneapolis, Minn, 1986), IMA Vol. Math. Appl., vol. 9, Springer, New York, 1987, pp. 75-97.

[7] T. Funaki and H. Spohn, Motion by mean curvature from the Ginzburg-Landau $\nabla \phi$ interface model, Communications in Mathematical Physics 185 (1997), no. 1, 1-36.

[8] M. Z. Guo, G. C. Papanicolaou, and S. R. S. Varadhan, Nonlinear diffusion limit for a system with nearest neighbor interactions, Communications in Mathematical Physics 118 (1988), no. 1, 31-59.

[9] C. Kipnis and C. Landim, Scaling Limits of Interacting Particle Systems, Fundamental Principles of Mathematical Sciences, vol. 320, Springer, Berlin, 1999.

[10] J. Krug, H. T. Dobbs, and S. Majaniemi, Adatom mobility for the solid-on-solid model, Zeitschrift für Physik B 97 (1995), no. 2, 281-291.

[11] C. Landim, S. Olla, and S. R. S. Varadhan, Symmetric simple exclusion process: regularity of the self-diffusion coefficient, Communications in Mathematical Physics 224 (2001), no. 1, 307-321.

[12] T. Nishikawa, Hydrodynamic limit for the Ginzburg-Landau $\nabla \phi$ interface model with a conservation law, Journal of Mathematical Sciences. The University of Tokyo 9 (2002), no. 3, 481-519.

[13] J. Quastel, Diffusion of color in the simple exclusion process, Communications on Pure and Applied Mathematics 45 (1992), no. 6, 623-679.

[14] L L Large deviations from a hydrodynamic scaling limit for a nongradient system, The Annals of Probability 23 (1995), no. 2, 724-742.

[15] _ Bulk diffusion in a system with site disorder, The Annals of Probability 34 (2006), no. 5. 
[16] A. Savu, Hydrodynamic scaling limit of continuum solid-on-solid model, Ph.D. thesis, University of Toronto, Toronto, 2004.

[17] Closed and exact functions in the context of Ginzburg-Landau models, to appear in Canadian Journal of Mathematics, http://arxiv.org/abs/math/0506002.

[18] M. Schimschak and J. Krug, Surface electromigration as a moving boundary value problem, Physical Review Letters 78 (1997), no. 2, 278-281.

[19] S. R. S. Varadhan, Nonlinear diffusion limit for a system with nearest neighbor interactions. II, Asymptotic Problems in Probability Theory: Stochastic Models and Diffusions on Fractals (Sanda/Kyoto, 1990), Pitman Res. Notes Math. Ser., vol. 283, Longman Sci. Tech., Harlow, 1993, pp. $75-128$.

[20] S. R. S. Varadhan and H.-T. Yau, Diffusive limit of lattice gas with mixing conditions, The Asian Journal of Mathematics 1 (1997), no. 4, 623-678.

Anamaria Savu: Department of Mathematics, University of Northern British Columbia, Prince George, BC, Canada V2N 4Z9

E-mail address: savua@unbc.ca 


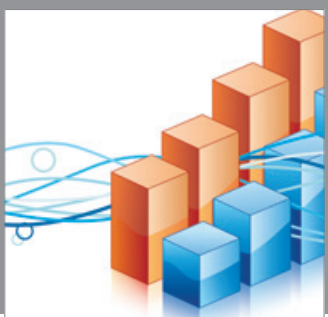

Advances in

Operations Research

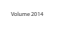

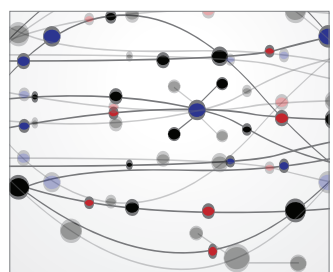

\section{The Scientific} World Journal
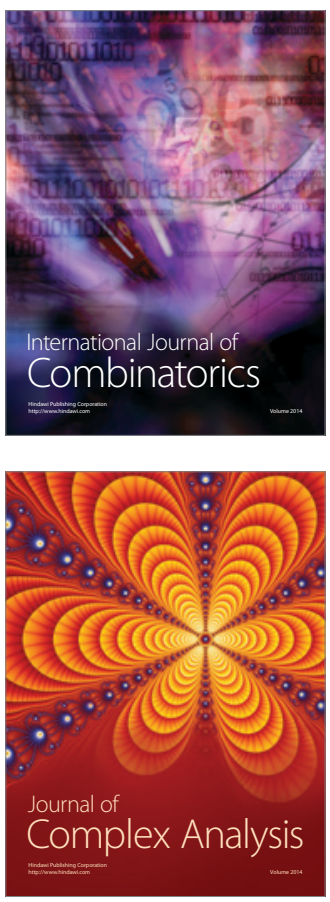

International Journal of

Mathematics and

Mathematical

Sciences
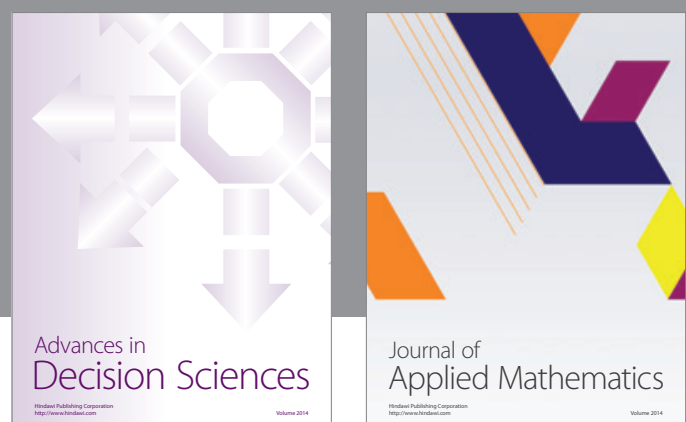

Journal of

Applied Mathematics
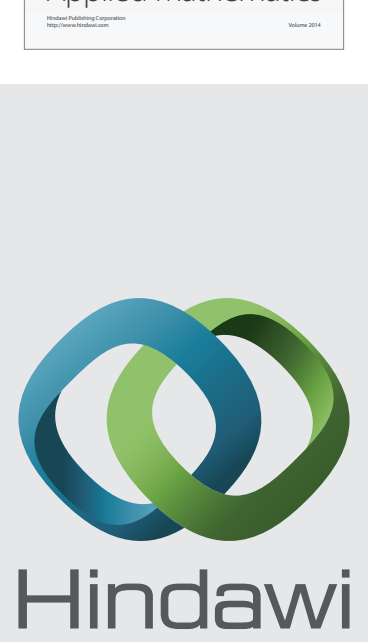

Submit your manuscripts at http://www.hindawi.com
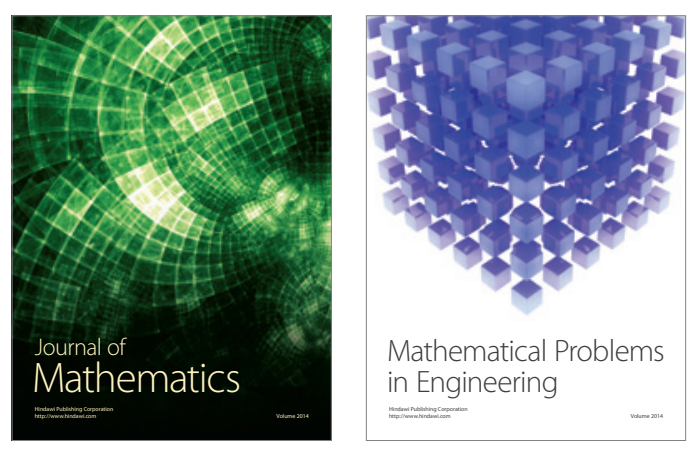

Mathematical Problems in Engineering
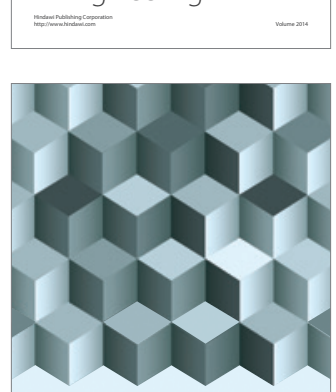

Journal of

Function Spaces
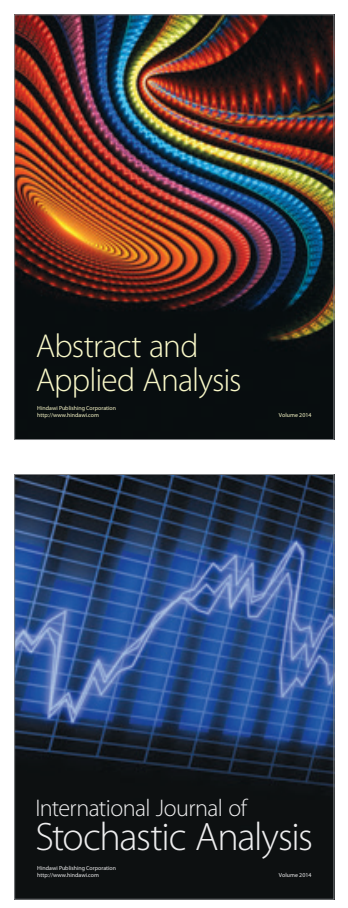

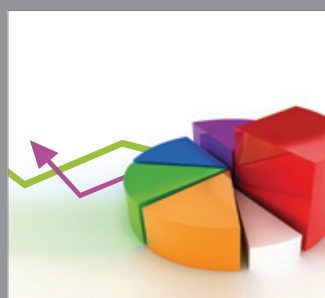

ournal of

Probability and Statistics

Promensencen
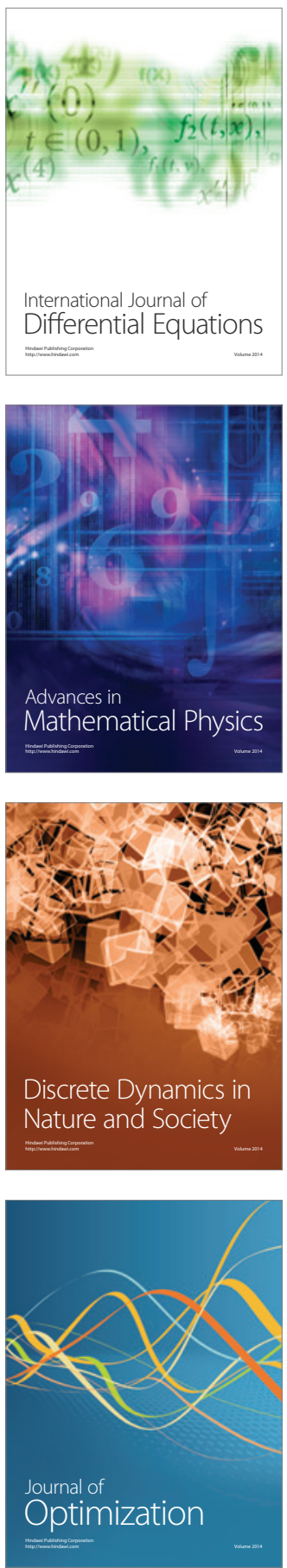